\title{
THEORY AND ALGORITHMS OF THE LAGUERRE TRANSFORM, PART II: ALGORITHM
}

\author{
Ushio Sumita \\ International University of Japan. \\ Masaaki Kijima \\ and \\ University of Rochester \\ University of Tsukuba
}

(Received September 14, 1990)

\begin{abstract}
The Laguerre transform developed by Keilson, Numn and Sumita (1979, 1981, 1981) provides an algorithmic framework for the computer evaluation of repeated combinations of various continuum operations such as multiple convolutions, integration, differentiation, and multiplication by polynomials. In the previous survey paper (1988), we summarize theoretical results on the Laguerre transform obtained up to date. Following the theoretical summary, the aim of this paper is to discuss the operational characters of the Laguerre transform when actually using it in applications. The two summary papers will enable the reader to use the Laguerre transform with ease.
\end{abstract}

\section{Introduction}

In the previous paper [A], a theoretical foundation was described of the Laguerre transform which maps a function $f(x)$ of $L_{2}$ into a discrete sequence $\left(f_{n}^{\#}\right)_{-\infty}^{\infty}$. Moreover, various continuum operations are mapped into lattice operations. One obtains thereby an algorithmic basis for computing multiple convolutions, integration, differentiation, multiplication by polynomials and related infinite series of importance to statistics and applied probability. As a sequel to the previous paper $[\mathrm{A}]$, this paper focuses on algorithmic aspects of the Laguerre transform.

One strong advantage of the Laguerre transform method in comparison with other conventional Laplace transform inversion techniques can be found in the general and systematic formalism built into its structure. The Laguerre sharp coefficients $\left(f_{n}^{\#}\right)_{-\infty}^{\infty}$ of $f(x)$ can be easily converted, via simple lattice operations, to those of e.g. $\int_{-\infty}^{\infty} f(x-y) f(y) d y, \int_{x}^{\infty} f(y) d y$, and $\frac{d}{d x} f(x)$, bypassing numerical integration and differentiation. Furthermore, the method enables one to perform a combination of these operations repeatedly without numerical difficulty, while numerical inversion of Laplace transforms often has to be done independently for each case.

Another factor that makes the Laguerre transform so attractive is the ability of representing functions to be manipulated by sequences of the Laguerre coefficients with efficiency and accuracy. For many functions of interest in applied probability and statistics, the Laguerre coefficients are available either analytically or via efficient numerical procedures. These include probability density functions in Exponential family, Gamma family, Logistic family, and Normal family.

Following the summary of the theoretical results of the Laguerre transform given in 
[A], this paper discusses the operational characters of the Laguerre transform from the point of view of actually using it in a variety of application contexts. Throughout the paper, references to formulas in [A] are indicated by (A.1.1.1), etc. It is hoped that this summary paper together with the previous paper $[\mathrm{A}]$ would help those who are interested in implementing the Laguerre transform method.

In Section 1, the key operational rules are described in a succinct and algorithm-oriented manner. Section 2 is devoted to the derivation of the Laguerre coefficients of basic probability density functions. Several bivariate exponential density functions are also discussed. In Section 3, we present some successful application of the Laguerre transform, newly reported here, in the dynamic waiting time behavior of an $M / G / 1$ queueing system with exhaustive server vacations.

\section{Algorithmic Description of Operational Rules of the Laguerre Transform}

Let $f(x)$ be a rapidly decreasing function in $C_{\downarrow}^{\infty *}(-\infty, \infty)$, as in (A.1.1.6), represented by the Laguerre dagger and sharp coefficient vectors

$$
\boldsymbol{f}^{\dagger}=\left(f_{-M}^{\dagger}, \cdots, f_{0}^{\dagger}, \cdots, f_{N}^{\dagger}\right)^{T} \text { and } \boldsymbol{f}^{\#}=\left(f_{-M}^{\#}, \cdots, f_{0}^{\#}, \cdots, f_{N}^{\#}\right)^{T},
$$

where $T$ denotes the transpose. The truncation points $M, N \geq 0$ are chosen so that the moment formulas satisfy a prespecified accuracy $\varepsilon>0$, see $\S 1.2$ of $[\mathrm{A}]$. For convenience, we denote the set of vectors whose components range from $-M$ to $N$ by $R(M, N)$. For $\boldsymbol{a}=\left(a_{n}\right) \in R(M, N), a_{n}$ is assumed to be zero for $n<-M$ and $n>N$. The purpose of this section is to describe several important continuum operations on $f(x)$ in terms of lattice operations on $\boldsymbol{f}^{\dagger}$ and $\boldsymbol{f}^{\#}$ in algorithmic forms. For other continuum operations, the reader is referred to $[\mathrm{A}]$ and $[11,12,20,33,38]$.

\subsection{Dagger/Sharp Coefficients and Representation of $f(x)$}

For a vector $\boldsymbol{a} \in R(M, N)$, we define the differencing operator

$$
\mathrm{DIFF}: R(M, N) \rightarrow R(M, N)
$$

and the partially summing operator

$$
\operatorname{PSUM}: R(M, N) \rightarrow R(M, N)
$$

by

$$
\boldsymbol{b}=\operatorname{DIFF}(\boldsymbol{a}) \Leftrightarrow b_{n}=\Delta\left[a_{n}\right]=a_{n}-a_{n-1},
$$

and

$$
\boldsymbol{b}=\operatorname{PSUM}(\boldsymbol{a}) \quad \Leftrightarrow \quad b_{n}=\sum_{m=-M}^{n} a_{m},
$$

respectively. As can be seen from (A.1.1.10), one then has

$$
f^{\#}=\operatorname{DIFF}\left(\boldsymbol{f}^{\dagger}\right) ; \quad \boldsymbol{f}^{\dagger}=\operatorname{PSUM}\left(\boldsymbol{f}^{\#}\right)
$$

Let

$$
\operatorname{INNP}: R(M, N) \times R(M, N) \rightarrow R
$$


be the inner product operator, i.e.

$$
\operatorname{INNP}(\boldsymbol{a}, \boldsymbol{b})=\sum_{n=-M}^{N} a_{n} b_{n}
$$

and define

$$
\boldsymbol{h}(x)=\left(h_{-M}(x), \cdots, h_{0}(x), \cdots, h_{N}(x)\right)^{T} .
$$

It should be noted from (A.1.1.2) that $h_{n}(x)=0$ for $-M \leq n<0$ if $x \geq 0$ and $h_{n}(x)=0$ for $0 \leq n \leq N$ if $x<0$. From (A.1.1.3) together with (1.1.3) through (1.1.5), it follows that

$$
f(x)=\operatorname{INNP}\left(\boldsymbol{f}^{\dagger}, \boldsymbol{h}(x)\right)=\operatorname{INNP}\left(\operatorname{PSUM}\left(\boldsymbol{f}^{\#}\right), \boldsymbol{h}(x)\right) .
$$

The values of $\boldsymbol{h}(x)$ can be generated efficiently based on the recursion formula (A.1.1.7). Hence, once $\boldsymbol{f}^{\dagger}$ or $\boldsymbol{f}^{\#}$ is obtained, the values of $f(x)$ can be computed easily via (1.1.6).

\subsection{Convolution and Integration}

For describing convolution and integration properties of the Laguerre transform, we introduce two lattice operators

$$
\text { CONV }: R(M, N) \times R(M, N) \rightarrow R(M, N)
$$

and

$$
\mathrm{SUV}: R(0, N) \rightarrow R(0, N)
$$

defined by

$$
\boldsymbol{c}=\operatorname{CONV}(\boldsymbol{a}, \boldsymbol{b}) \Leftrightarrow c_{n}=\sum_{m=-M}^{N} a_{n-m} b_{m}
$$

and

$$
\boldsymbol{b}=\operatorname{SUV}(\boldsymbol{a}) \Leftrightarrow b_{n}=-2 a_{n}+4 \sum_{m=0}^{N}(-1)^{m} a_{m+1+n},
$$

respectively. It then follows from Theorem $1.3 \mathrm{~B}$ of $[\mathrm{A}]$ that

$$
r(x)=f * g(x)=\int_{-\infty}^{\infty} f(x-y) g(y) d y \quad \Leftrightarrow \quad r^{\#}=\operatorname{CONV}\left(f^{\#}, g^{\#}\right)
$$

and

$$
r(x)=\int_{x}^{\infty} f(x) d y \quad(f \text { on }[0, \infty)) \quad \Leftrightarrow \quad \boldsymbol{r}^{\#}=\operatorname{SUV}\left(\boldsymbol{f}^{\#}\right)
$$

We recall that the tail integral $\int_{x}^{\infty} f(x) d x$ for $f$ on $(-\infty, \infty)$ can be treated based on (1.2.4), see Remark 1.3C of [A].

For treating multiple convolutions, we define

$$
\operatorname{CONV}_{i=1}^{K}\left(\boldsymbol{a}_{i}\right)=\operatorname{CONV}\left(\operatorname{CONV}_{i=1}^{K-1}\left(\boldsymbol{a}_{i}\right), \boldsymbol{a}_{K}\right)
$$

with $\operatorname{CONV}_{i=1}^{1}\left(a_{i}\right)=a_{1}$. Then,

$$
r(x)=f_{1} * \cdots * f_{n}(x) \quad \Leftrightarrow \quad \boldsymbol{r}^{\#}=\operatorname{CONV}_{i=1}^{n}\left(f_{i}^{\#}\right) .
$$




\subsection{Shifting and Mixing}

As the shifting operator, we define

$$
\mathrm{SHIF}: R(M, N) \times R \rightarrow R(M, N)
$$

by

$$
\operatorname{SHIF}(\boldsymbol{a}, T)=\operatorname{CONV}(\boldsymbol{a}, \Delta[\boldsymbol{h}(T)]) .
$$

Then, from Theorem 1.3G(a) of $[\mathrm{A}]$,

$$
r(x)=f(x-T) \quad \Leftrightarrow \quad \boldsymbol{r}^{\#}=\operatorname{SHIF}\left(\boldsymbol{f}^{\#}, T\right) .
$$

Also, as the mixing operator

$$
\text { MIX }: R^{K} \times R(M, N)^{K} \rightarrow R(M, N),
$$

we introduce

$$
\operatorname{MIX}\left(p_{1}, \cdots, p_{K} ; \boldsymbol{a}_{1}, \cdots, \boldsymbol{a}_{K}\right)=\sum_{j=1}^{K} p_{j} \boldsymbol{a}_{j} .
$$

Since the Laguerre transform is linear, one then has

$$
r(x)=\sum_{j=1}^{K} p_{j} f_{j}(x) \Leftrightarrow\left\{\begin{array}{l}
\boldsymbol{r}^{\dagger}=\operatorname{MIX}\left(p_{1}, \cdots, p_{K} ; \boldsymbol{f}_{1}^{\dagger}, \cdots, \boldsymbol{f}_{K}^{\dagger}\right), \\
\boldsymbol{r}^{\#}=\operatorname{MIX}\left(p_{1}, \cdots, p_{K} ; \boldsymbol{f}_{1}^{\#}, \cdots, \boldsymbol{f}_{K}^{\#}\right) .
\end{array}\right.
$$

\subsection{Differentiation and Multiplication by Polynomial}

For differentiation property of the Laguerre transform, we introduce the lattice operator

$$
\mathrm{DER}: R(M, N) \rightarrow R(M, N)
$$

defined by

$$
\boldsymbol{b}=\operatorname{DER}(\boldsymbol{a}) \quad \Leftrightarrow \quad b_{n}=\frac{1}{2}\left(a_{n}+a_{n-1}\right) .
$$

Let $f(x)$ be differentiable except $x=0 . \quad f(x)$ may have discrepancy at the origin. Let $\delta \in R(M, N)$ be such that $\delta_{n}=1$ for $n=0$ and $\delta_{n}=0$ elsewhere. Then, from Theorem $1.3 \mathrm{D}$ of $[\mathrm{A}]$, one has

$$
r(x)=\frac{d}{d x} f(x) \quad(x \neq 0) \quad \Leftrightarrow \quad r^{\#}=\operatorname{DER}\left(f^{\dagger}\right)+\left.\delta f(x)\right|_{x=0-} ^{0+} .
$$

We note that, for $f$ on $[0, \infty)$, the operators SUV in (1.2.2) and DER in (1.4.2) are in a dual relation in the sense that $\boldsymbol{f}^{\#}=-\operatorname{DER}\left(\operatorname{PSUM}\left(\operatorname{SUV}\left(\boldsymbol{f}^{\#}\right)\right)\right)$.

To describe the operational property of multiplication by polynomial, we define the operator

$$
\text { MUL }: R(M, N) \rightarrow R(M, N)
$$

by

$$
\boldsymbol{b}=\operatorname{MUL}(\boldsymbol{a}) \Leftrightarrow b_{n}=-\Delta^{2}\left[(n+1) a_{n+1}\right],
$$


where $\Delta^{2}\left[a_{n}\right]=\Delta\left[\Delta\left[a_{n}\right]\right]$. The corresponding $k$-fold operator is defined as

$$
\boldsymbol{b}=\mathrm{MUL}_{k}(\boldsymbol{a})=\mathrm{MUL}_{k-1}(\operatorname{MUL}(\boldsymbol{a}))
$$

with $\mathrm{MUL}_{1}=\mathrm{MUL}$. It then follows from Theorem 1.3E of $[\mathrm{A}]$ that

$$
r(x)=x^{k} f(x) \quad \Leftrightarrow \quad r^{\#}=\operatorname{MUL}_{k}\left(f^{\#}\right) .
$$

A combination of (1.3.4) and (1.4.5) then provides the operational property of multiplication by polynomial of the Laguerre transform.

\subsection{Product of Functions}

Let $R\left(M_{1}, M_{2} ; N_{1}, N_{2}\right)$ be the set of matrices whose first components range from $-M_{1}$ to $M_{2}$ and second from $-N_{1}$ to $N_{2}$. The matrix version of the conventions used in the previous section will be also used unless confusions occur. For matrices $B=\left(b_{m n}\right)$ and $C=\left(c_{m n}\right)$ in $R\left(M_{1}, M_{2} ; N_{1}, N_{2}\right)$, define the bivariate inner product operator

$$
\text { BINNP : } R\left(M_{1}, M_{2} ; N_{1}, N_{2}\right) \times R\left(M_{1}, M_{2} ; N_{1}, N_{2}\right) \rightarrow R
$$

by

$$
\operatorname{BINNP}(\boldsymbol{B}, \boldsymbol{C})=\sum_{m=-M_{1}}^{M_{2}} \sum_{n=-N_{1}}^{N_{2}} b_{m n} c_{m n}
$$

For vectors $\boldsymbol{a} \in R\left(M_{1}, M_{2}\right)$ and $\boldsymbol{b} \in R\left(N_{1}, N_{2}\right)$, we define the outer product operator

$$
\text { OUTP : } R\left(M_{1}, M_{2}\right) \times R\left(N_{1}, N_{2}\right) \rightarrow R\left(M_{1}, M_{2} ; N_{1} \cdot N_{2}\right)
$$

by

$$
\boldsymbol{C}=\operatorname{OUTP}(\boldsymbol{a}, \boldsymbol{b}) \quad \Leftrightarrow \quad c_{m n}=a_{m} b_{n} .
$$

Now, denoting the Laguerre functions by $\ell_{n}(x)$, let $a_{i j}(n)=\int_{0}^{\infty} \ell_{i}(x) \ell_{j}(x) \ell_{n}(x) d x$ and construct the matrix $\boldsymbol{A}(n)=\left(a_{i j}(n)\right) \in R(0, M ; 0, N)$. Also, define the product operator

$$
\text { PROD }: R(0, M ; 0, N) \rightarrow R(0, \min \{M, N\})
$$

by

$$
\boldsymbol{c}=\operatorname{PROD}(\boldsymbol{B}) \quad \Leftrightarrow \quad c_{n}=\operatorname{BINNP}(\boldsymbol{B}, \boldsymbol{A}(n)) .
$$

Then, from Theorem 1.3I of [A],

$$
r(x)=f(x) g(x) \quad(f, g \text { on }[0, \infty)) \quad \Leftrightarrow \quad \boldsymbol{r}^{\dagger}=\operatorname{PROD}\left(\operatorname{OUTP}\left(\boldsymbol{f}^{\dagger}, \boldsymbol{g}^{\dagger}\right)\right) .
$$

The calculation of $\boldsymbol{A}(n)$ requires more explanation (see [20,40] for the theoretical foundation). For convenience, it is assumed that $M \leq N$. Let $\boldsymbol{V}$ be the $(M+1) \times(M+1)$ symmetric tri-diagonal matrix defined by

$$
\boldsymbol{V}=\left(\begin{array}{ccccc}
1 & -1 & & & \\
-1 & 3 & -2 & & \\
& \ddots & \ddots & \ddots & \\
& & -M+1 & 2 M-1 & -M \\
& & & -M & 2 M+1
\end{array}\right)
$$


The matrix $\boldsymbol{V}$ is the principal submatrix of the Q-matrix governing linear growth birth-death processes $[8,9]$. Let $\boldsymbol{A}(0)=\left(a_{i j}(0)\right)$ be such that

$$
a_{i j}(0)=2 \sum_{k=\max \{i, j\}}^{i+j} t(i, j ; k) ; \quad t(i, j ; k)=\frac{1}{3^{k+1}}(k)\left(k \underline{i}_{j} j\right) .
$$

The matrices $\boldsymbol{A}(n)$ are then successively generated by

$$
\boldsymbol{A}(n+1)=\frac{1}{n+1}[\{(2 n+1) \boldsymbol{I}-\boldsymbol{V}\} \boldsymbol{A}(n)-n \boldsymbol{A}(n-1)], \quad 0 \leq n \leq M
$$

with $\boldsymbol{A}(-1)$ being the zero matrix.

\subsection{The Bivariate Laguerre Transform}

Thus far, we have described, in algorithmic forms, lattice operators of importance for the practical use of the univariate Laguerre transform. For the bivariate Laguerre transform, such descriptions are also possible by following the manners in Sections 1.1 through 1.5. Here, however, to avoid inessential repetitions, we describe only the bivariate lattice operators that will be used in the subsequent sections.

Let $f(x, y)$ be a rapidly decreasing function equipped with the Laguerre dagger and sharp coefficient matrices $\boldsymbol{F}^{\dagger}=\left(f_{m n}^{\dagger}\right)$ and $\boldsymbol{F}^{\#}=\left(f_{m n}^{\#}\right)$ in $R\left(M_{1}, M_{2} ; N_{1}, N_{2}\right)$. As for the univariate case, the truncation points $M_{1}, M_{2}, N_{1}$ and $N_{2} \geq 0$ are chosen so that the moment formulas satisfy a prespecified accuracy, see $\S 2.2$ of [A].

For a matrix $\boldsymbol{A} \in R\left(M_{1}, M_{2} ; N_{1}, N_{2}\right)$, define the operators

$$
\mathrm{BDIFF}: R\left(M_{1}, M_{2} ; N_{1}, N_{2}\right) \rightarrow R\left(M_{1}, M_{2} ; N_{1}, N_{2}\right)
$$

and

$$
\mathrm{BPSUM}: R\left(M_{1}, M_{2} ; N_{1}, N_{2}\right) \rightarrow R\left(M_{1}, M_{2} ; N_{1}, N_{2}\right)
$$

by

$$
\boldsymbol{B}=\operatorname{BDIFF}(\boldsymbol{A}) \Leftrightarrow b_{m n}=\Delta\left[a_{m n}\right]=\Delta_{1}\left[\Delta_{2}\left[a_{m n}\right]\right],
$$

where $\Delta_{1}\left[a_{m n}\right]=a_{m n}-a_{m-1, n}$ and $\Delta_{2}\left[a_{m n}\right]=a_{m n}-a_{m, n-1}$, and

$$
\boldsymbol{B}=\operatorname{BPSUM}(\boldsymbol{A}) \quad \Leftrightarrow \quad b_{m n}=\sum_{i=-M_{1}}^{m} \sum_{j=-N_{1}}^{n} a_{i j},
$$

respectively. Then, as for the univariate case, one has from (A.2.1.12)

$$
\boldsymbol{F}^{\#}=\operatorname{BDIFF}\left(\boldsymbol{F}^{\dagger}\right) ; \quad \boldsymbol{F}^{\dagger}=\operatorname{BPSUM}\left(\boldsymbol{F}^{\#}\right)
$$

Let $\boldsymbol{H}(x)=\left(h_{m n}(x)\right)=\operatorname{OUTP}(\boldsymbol{h}(x), \boldsymbol{h}(x)) \in R\left(M_{1}, M_{2} ; N_{1}, N_{2}\right)$. The series representation (A.2.1.3) is then written by a convenient form as

$$
f(x, y)=\operatorname{BINNP}\left(\boldsymbol{F}^{\dagger}, \boldsymbol{H}(x)\right)=\operatorname{BINNP}\left(\operatorname{BPSUM}\left(\boldsymbol{F}^{\#}\right), \boldsymbol{H}(x)\right) .
$$

Hence, once $\boldsymbol{F}^{\dagger}$ or $\boldsymbol{F}^{\#}$ is given, the values of $f(x, y)$ are readily computed via (1.6.4). 
For describing typical convolution properties of the bivariate Laguerre transform, we introduce the bivariate lattice operators

$$
\begin{gathered}
\mathrm{BCONV}: R\left(M_{1}, M_{2} ; N_{1}, N_{2}\right) \times R\left(M_{1}, M_{2} ; N_{1}, N_{2}\right) \rightarrow R\left(M_{1}, M_{2} ; N_{1}, N_{2}\right), \\
\operatorname{MCONV}: R\left(M_{1}, M_{2}\right) \times R\left(M_{1}, M_{2} ; N_{1}, N_{2}\right) \rightarrow R\left(M_{1}, M_{2} ; N_{1}, N_{2}\right),
\end{gathered}
$$

and

$$
\mathrm{SCONV}: R\left(M_{1}, M_{2} ; N_{1}, N_{2}\right) \rightarrow R\left(\min \left\{M_{1}, N_{1}\right\}, \min \left\{M_{2}, N_{2}\right\}\right)
$$

defined by

$$
\begin{gathered}
\boldsymbol{C}=\operatorname{BCONV}(\boldsymbol{A}, \boldsymbol{B}) \Leftrightarrow c_{m n}=\sum_{i=-M_{1}}^{M_{2}} \sum_{j=-N_{1}}^{N_{2}} a_{m-i, n-j} b_{i j}, \\
\boldsymbol{C}=\operatorname{MCONV}(\boldsymbol{a}, \boldsymbol{B}) \Leftrightarrow c_{m n}=\sum_{k=-M_{1}}^{M_{2}} a_{m-k} b_{k n}
\end{gathered}
$$

and

$$
\boldsymbol{b}=\operatorname{SCONV}(\boldsymbol{A}) \Leftrightarrow b_{m}=\sum_{n=-\min \left\{M_{1}, N_{1}\right\}}^{\min \left\{M_{2}, N_{2}\right\}} a_{m-n, n} .
$$

Accordingly, from Theorem 2.3B of [A], one has

$$
\begin{gathered}
r(x, y)=f * g(x, y)=\int_{-\infty}^{\infty} \int_{-\infty}^{\infty} f\left(x-x^{\prime}, y-y^{\prime}\right) g\left(x^{\prime}, y^{\prime}\right) d x^{\prime} d y^{\prime} \\
\Leftrightarrow \quad \boldsymbol{R}^{\#}=\operatorname{BCONV}\left(\boldsymbol{F}^{\#}, \boldsymbol{G}^{\#}\right) \\
r(x, y)=f *_{1} g(x, y)=\int_{-\infty}^{\infty} f\left(x-x^{\prime}\right) g\left(x^{\prime}, y\right) d x^{\prime} \quad\left(f \in C_{\downarrow}^{\infty *}(-\infty, \infty)\right) \\
\Leftrightarrow \quad \boldsymbol{R}^{\#}=\operatorname{MCONV}\left(\boldsymbol{f}^{\#}, \boldsymbol{G}^{\#}\right)
\end{gathered}
$$

and

$$
r(x)=\int_{-\infty}^{\infty} f(x-y, y) d y \quad \Leftrightarrow \quad \boldsymbol{r}^{\#}=\operatorname{SCONV}\left(\boldsymbol{F}^{\#}\right)
$$

Note that the marginal convolution with respect to the second component in (1.6.9) can be performed as

$$
\boldsymbol{R}^{\#}=\operatorname{MCONV}\left(\boldsymbol{f}^{\#}, \boldsymbol{G}^{\# T}\right)^{T} .
$$

Finally, we give the lattice operators associated with integration. Let

$$
\begin{gathered}
\text { MARG }: R\left(M_{1}, M_{2} ; N_{1}, N_{2}\right) \rightarrow R\left(M_{1}, M_{2}\right), \\
\text { MSUV }: R(0, M ; 0, N) \rightarrow R(0, M ; 0, N),
\end{gathered}
$$

and

$$
\text { BSUV }: R(0, M ; 0, N) \rightarrow R(0, M ; 0, N)
$$

be defined by

$$
\boldsymbol{b}=\operatorname{MARG}(\boldsymbol{A}) \Leftrightarrow b_{n}=\sum_{n=-N_{1}}^{N_{2}}(-1)^{n} a_{m n},
$$




$$
\boldsymbol{B}=\operatorname{MSUV}(\boldsymbol{A}) \quad \Leftrightarrow \quad b_{m n}=2 \sum_{i=m+1}^{M} \sum_{j=0}^{n}(-1)^{m+i} a_{i j}
$$

and

$$
\boldsymbol{B}=\operatorname{BSUV}(\boldsymbol{A}) \Leftrightarrow b_{m n}=4 \sum_{i=m+1}^{M} \sum_{j=n+1}^{N}(-1)^{m+n+i+j} a_{i j},
$$

respectively. The marginal integration is then reduced to, from Theorem $2.3 \mathrm{C}$ of $[\mathrm{A}]$,

$$
r(x)=\int_{-\infty}^{\infty} f(x, y) d y \quad \Leftrightarrow \quad \boldsymbol{r}^{\#}=\operatorname{MARG}\left(\boldsymbol{F}^{\#}\right)
$$

For the marginal integration with respect to $x$, the transposed $\boldsymbol{F}^{\#}$ is employed in (1.6.15). Also, from Theorem 2.3D of $[\mathrm{A}]$, for $f$ on $[0, \infty) \times[0, \infty)$,

$$
r(x, y)=\int_{x}^{\infty} f\left(x^{\prime}, y\right) d x^{\prime} \Leftrightarrow \boldsymbol{R}^{\#}=\operatorname{MSUV}\left(\boldsymbol{F}^{\#}\right)
$$

and

$$
r(x, y)=\int_{y}^{\infty} \int_{x}^{\infty} f\left(x^{\prime}, y^{\prime}\right) d x^{\prime} d y^{\prime} \Leftrightarrow \boldsymbol{R}^{\#}=\operatorname{BSUV}\left(\boldsymbol{F}^{\#}\right)
$$

For further discussions of the operational properties in the bivariate Laguerre transform, the reader is referred to $[20,38]$.

\section{The Laguerre Coefficients of Functions of Interest in Applied Probability and Statistics}

Applicability of the Laguerre transform method hinges on finding the Laguerre coefficients of functions to be manipulated with speed and accuracy. In this section, we first describe several general procedures for accomplishing this objective. In some applications, two functions on $[0, \infty)$ have to be patched together to construct one function on $(-\infty, \infty)$. We also discuss how this task can be done via the Laguerre transform. In the remaining parts of this section, these ideas are employed in order to derive the Laguerre coefficients of functions of interest in applied probability and statistics.

\subsection{General Procedures for Finding the Laguerre Coefficients}

In the Laguerre transform, the inversion difficulty in the Laplace or Fourier transform is replaced by the problem of finding the Laguerre sharp coefficients

$$
f_{n}^{\#}=\int_{-\infty}^{\infty} f(x) \Delta\left[h_{n}(x)\right] d x, \quad-M \leq n \leq N .
$$

Once the coefficient vector $f^{\#}$ is found, as we saw in Section 1 , most of basic continuum operations are mapped into lattice operations and the inversion procedure back onto continuum is straightforward. We next present several general approaches for finding the Laguerre coefficients.

\subsection{A Generating Function Approach}


Based on the key formula $T_{f}^{\#}(u)=\phi_{f}\left(\frac{1}{2} \frac{1+u}{1-u}\right)$ as given in (A.1.1.12), find $f_{n}^{\#}$ by expanding $\phi_{f}\left(\frac{1}{2} \frac{1+u}{1-u}\right)$ as a power of $u$.

\subsection{B Recursion Formula Approach}

Find the first order differential equation of $\phi(s)$ of the form

$$
v(s) \frac{d}{d s} \phi(s)+w(s) \phi(s)+z(s)=0 .
$$

By substituting $s=(1+u) / 2(1-u)$ into (2.1.2), one has

$$
v\left(\frac{1}{2} \frac{1+u}{1-u}\right)(1-u)^{2} \frac{d}{d u} T_{f}^{\#}(u)+w\left(\frac{1}{2} \frac{1+u}{1-u}\right) T_{f}^{\#}(u)+z\left(\frac{1}{2} \frac{1+u}{1-u}\right)=0 .
$$

Using (2.1.3), a recursion formula on $f_{n}^{\#}$ may be derived. Since $T_{f}^{\#}(u)=(1-u) T_{f}^{\dagger}(u)$, Equation (2.1.3) can be rewritten as

$$
\begin{aligned}
& v\left(\frac{1}{2} \frac{1+u}{1-u}\right)(1-u)^{3} \frac{d}{d u} T_{f}^{\dagger}(u)+ \\
& \left\{w\left(\frac{1}{2} \frac{1+u}{1-u}\right)-v\left(\frac{1}{2} \frac{1+u}{1-u}\right)(1-u)\right\}(1-u) T_{f}^{\dagger}(u)+z\left(\frac{1}{2} \frac{1+u}{1-u}\right)=0,
\end{aligned}
$$

which enables one to convert the recursion formula on $f_{n}^{\#}$ to that on $f_{n}^{\dagger}$.

\subsection{Fast Fourier Transform Approach}

The third approach is to approximate $T_{f}^{\#}(u)$ by

$$
\tilde{T}_{f}^{\#}(u)=\sum_{n=-M}^{M} f_{n}^{\#} u^{n} .
$$

From the key formula (A.1.1.12), one sees that $T_{f}^{\#}\left(e^{i \theta}\right)=\phi_{f}\left(\frac{i}{2} \cot \frac{\theta}{2}\right)$. Based on the approximation in (2.1.5), one then obtains a set of linear equations

$$
W f^{\#}=\boldsymbol{\beta}
$$

where

$$
\begin{gathered}
\boldsymbol{W}=\left(w_{r n}\right) ; \quad w_{r n}=e^{i n \theta(r)} ; \quad \theta(r)=\frac{2 \pi r}{2 M}, \quad r=0, \cdots 2 M, \\
f^{\#}=\left(f_{-M}^{\#}, \cdots, f_{0}^{\#}, \cdots, f_{M}^{\#}\right)^{T}
\end{gathered}
$$

and

$$
\boldsymbol{\beta}=\left(\beta_{0}, \cdots, \beta_{2 M}\right)^{T} ; \quad \beta_{r}=\phi_{f}\left(\frac{i}{2} \cot \frac{\theta(r)}{2}\right) .
$$

Because of the simple structure of $\boldsymbol{W}$, one has $\left(\boldsymbol{W}^{-1}\right)_{r n}=1 /\left(2 w_{r n} M\right)$ and $(2.1 .6)$ can be solved for $\boldsymbol{f}^{\#}$. The straightforward way of obtaining $f^{\#}$ via (2.1.6) has complexity of $O\left(M^{2}\right)$. The fast Fourier transform, however, accomplishes this task with reduced complexity of $O(M \log M)$, see Sumita [33]. 


\subsection{Construction of Functions on $(-\infty, \infty)$ from Functions on $[0, \infty)$ via the Laguerre Transform}

In some applications, one encounters a function $f(x)$ on $(-\infty, \infty)$ constructed from two functions on $[0, \infty)$. More specifically, let $U(x)=1, x \geq 0$, and $U(x)=0, x<0$, and define

$$
f(x)=g(x) U(x)+r(-x) U(-x)
$$

where both $g(x)$ and $r(x)$ are functions on $[0, \infty)$. From the definition of $h_{n}(x)$ in (A.1.1.2) together with (A.1.1.3), the Laguerre dagger coefficients $f_{n}^{\dagger}$ of $f(x)$ are obtained from $g_{n}^{\dagger}$ and $r_{n}^{\dagger}$ of $g(x)$ and $r(x)$, respectively, by

$$
f_{n}^{\dagger}=g_{n}^{\dagger}, \quad n \geq 0 ; \quad f_{n}^{\dagger}=-r_{-n}^{\dagger}, \quad n<0 .
$$

Let

$$
\mathrm{CJCT}: R(0, M) \times R(0, N) \rightarrow R(M, N)
$$

be the lattice operator defined by

$$
\boldsymbol{c}=\operatorname{CJCT}(\boldsymbol{a}, \boldsymbol{b}) \Leftrightarrow \quad c_{n}= \begin{cases}a_{n}, & n>0, \\ a_{0}+b_{0}, & n=0, \\ b_{-n}, & n<0 .\end{cases}
$$

Then, one has from (2.2.2) and (A.1.1.10),

$$
\boldsymbol{f}^{\#}=\operatorname{CJCT}\left(\boldsymbol{g}^{\#}, \boldsymbol{r}^{\#}\right) \text {. }
$$

Based on (2.2.4), a variety of two sided functions can be constructed from one sided functions. We will see, for example, a Laplace-like probability density function (p.d.f.) can be constructed from two exponential p.d.f.'s.

\subsection{Exponential Family}

The power of the Laguerre transform appears mainly due to the fact that the Laguerre coefficients are found either analytically or via efficient numerical procedures. Especially, the Laguerre coefficients for exponential functions are given by a simple closed form. Exponential distributions are important not only because they themselves often appear in applied probability but also any p.d.f. can be arbitrarily approximated by multiple convolutions of exponential p.d.f.s and mixtures of the resulting p.d.f.s. The class of such distributions is often called the phase type distribution [30]. As we have already seen, the multiple convolution and mixing operations of the Laguerre transform are performed with ease via (1.2.5) and (1.3.4), respectively. Hence, in principle, the Laguerre coefficients of any p.d.f. can be found based on the Laguerre coefficients of exponential distributions. In this subsection, we summarize the way of finding the Laguerre coefficients of p.d.f.s in the exponential family.

\subsection{A Exponential Density}

For an exponential distribution having the mean $\theta^{-1}$, we denote, throughout the paper, its p.d.f. by

$$
e(\theta ; x)=\theta e^{-\theta x}, \quad x \geq 0, \quad \theta>0 .
$$


The Laplace transform of $e(\theta, x)$ is given by

$$
\phi_{f}(s)=\frac{\theta}{\theta+s}, \quad R e(s)>-\theta .
$$

Let $\left(e_{n}^{\dagger}(\theta)\right)_{0}^{\infty}$ be the dagger coefficients of $e(\theta ; x)$. Then, from the key formula (A.1.1.12) and the fact (A.1.1.9), one has

$$
T_{e}^{\dagger}(u)=\frac{2 \theta}{(2 \theta+1)-(2 \theta-1) u}=\frac{2 \theta}{2 \theta+1} \sum_{n=0}^{\infty}\left(\frac{2 \theta-1}{2 \theta+1}\right)^{n} u^{n}, \quad|u|<1 .
$$

Hence, comparing the coefficient of $u^{n}$ (see $\S 2.1 \mathrm{~A}$ ), it follows that

$$
e_{n}^{\dagger}(\theta)=\frac{2 \theta}{2 \theta+1}\left(\frac{2 \theta-1}{2 \theta+1}\right)^{n}, \quad n \geq 0
$$

Let

$$
\operatorname{EXP}: R \rightarrow R(0, N)
$$

be the lattice operator defined by

$$
\boldsymbol{a}=\operatorname{EXP}(\theta) \quad \Leftrightarrow \quad a_{n}=\frac{2 \theta}{2 \theta+1}\left(\frac{2 \theta-1}{2 \theta+1}\right)^{n} .
$$

The Laguerre coefficients of the exponential density $e(\theta ; x)$ are then obtained as

$$
\boldsymbol{e}^{\dagger}(\theta)=\operatorname{EXP}(\theta) ; \quad \boldsymbol{e}^{\#}(\theta)=\operatorname{DIFF}(\operatorname{EXP}(\theta))
$$

Remark 2.3.1. Let $f_{c}(x)=c e(\theta ; c x)$. The Laguerre dagger coefficients $\left(f_{c: n}^{\dagger}\right)_{0}^{\infty}$ of $f_{c}(x)$ are obtained by replacing $\theta$ with $c \theta$ in (2.3.3). It is easy to see that, if $c \theta=1 / 2$, then $f_{c: 0}^{\dagger}=1 / 2$ and $f_{c: n}^{\dagger}=0$ for $n \geq 1$. The tail of the sequence $\left(f_{c: n}^{\dagger}\right)_{0}^{\infty}$ quickly disappears if $c \theta$ is near $1 / 2$. The sequence has a rather long tail if $c \theta$ is either too large or too small. Hence it is desired to choose $c$, in calculating the Laguerre coefficients of an exponential density, so that $c \theta$ is neither too large nor too small. The scaling manipulation of this sort often solves the storage problem in computers.

\subsection{B Completely Monotone Density}

Let $G(x)$ be a distribution function defined on $[0, \infty)$. A p.d.f. $f(x)$ is said to be completely monotone if its Laplace transform is given by $\phi_{f}(s)=\int_{0}^{\infty} \frac{\theta}{\theta+s} d G(\theta)$, see e.g. Widder [50] and Feller [4]. If $G(x)$ consists of finitely many discrete spectrums, one has

$$
f(x)=\sum_{j=1}^{K} p_{j} e\left(\theta_{j} ; x\right), \quad p_{j}>0, \quad \sum_{j=1}^{K} p_{j}=1 .
$$

Hence, from (1.3.4) and (2.3.5),

$$
\boldsymbol{f}^{\dagger}=\operatorname{MIX}\left(p_{1}, \cdots, p_{K} ; \boldsymbol{e}^{\dagger}\left(\theta_{1}\right), \cdots, \boldsymbol{e}^{\dagger}\left(\theta_{K}\right)\right)
$$

\subsection{PF Density}


Let $f(x)$ be a p.d.f. whose Laplace transform is given as

$$
\phi_{f}(s)=\prod_{j=1}^{K} \frac{\theta_{j}}{\theta_{j}+s}
$$

i.e. $f(x)$ is a finite convolution of exponential densities $e\left(\theta_{j} ; x\right)$. It is known that $f(x)$ is Polyá-frequency of infinite order (see Karlin [7] and Keilson [10]). The sharp coefficients of $f(x)$ is obtained from $(1.2 .5)$ as

$$
f^{\#}=\operatorname{CONV}_{i=1}^{K}\left(e^{\#}\left(\theta_{i}\right)\right)
$$

\subsection{Phase Type Density}

A useful and important class of univariate distributions is the class of phase type distributions, see Neuts [30]. This class is known to be dense in the class of all one-sided proper distributions. Let $f(x)$ be the density function of a phase type distribution. The density function is given as a finite mixture of finite convolutions of exponential densities. In other words, the Laplace transform $\phi_{f}(s)$ of $f(x)$ is given as

$$
\phi_{f}(s)=\sum_{j=1}^{K} p_{j} \prod_{m=1}^{M_{j}} \frac{\theta_{m j}}{\theta_{m j}+s}, \quad K, M_{j} \geq 1
$$

where $p_{j}>0$ with $\sum_{j=1}^{K} p_{j}=1$. From (2.3.6) and (2.3.8), it is easy to see that

$$
f^{\#}=\operatorname{MIX}\left(p_{1}, \cdots, p_{K} ; \operatorname{CONV}_{i=1}^{M_{1}}\left(e^{\#}\left(\theta_{i 1}\right)\right), \cdots, \operatorname{CONV}_{i=1}^{M_{K}}\left(e^{\#}\left(\theta_{i K}\right)\right)\right) .
$$

\subsection{E Generalized Phase Type Density}

Let $f(x)$ be a generalized p.d.f. whose Laplace transform is given as

$$
\phi_{f}(s)=\sum_{j=0}^{\infty} p_{j}\left(\frac{\theta}{\theta+s}\right)^{j}, \quad \theta>0, p_{j} \geq 0, \sum_{j=0}^{\infty} p_{j}=1 .
$$

If $p_{0}>0, f(x)$ includes the delta function $p_{0} \delta(x)$. This $f(x)$ is called a generalized phase type distribution, see Shanthikumar [31]. It is known that the class of generalized phase type distributions is broader than the class of phase type distributions and is closed under convolution, mixture and formation of coherent system. The Laguerre transform of generalized phase type distributions has been developed in Shanthikumar [31]. In practice, using an appropriate truncation, say $K$, the sharp coefficients are obtained as

$$
f^{\#}=\operatorname{MIX}\left(p_{1}, \cdots, p_{K} ; e^{\#}(\theta), \operatorname{CONV}_{i=1}^{2}\left(e^{\#}(\theta)\right), \cdots, \operatorname{CONV}_{i=1}^{K}\left(e^{\#}(\theta)\right)\right) .
$$

\subsection{F Laplace-like Density}

In the notation $(2.2 .1)$, let $g(x)=\frac{\beta}{\alpha+\beta} e(\alpha ; x), x \geq 0$, and let $r(x)=\frac{\alpha}{\alpha+\beta} e(\beta ; x), x \geq 0$. The Laplace-like density $f(x)$ is written as

$$
f(x)= \begin{cases}\frac{\alpha \beta}{\alpha+\beta} e^{-\alpha x}, & x \geq 0, \\ \frac{\alpha \beta}{\alpha+\beta} e^{\beta x}, & x<0 .\end{cases}
$$

From (2.2.4), one then has

$$
f^{\#}=\operatorname{CJCT}\left(\frac{\beta}{\alpha+\beta} e^{\#}(\alpha), \frac{\alpha}{\alpha+\beta} e^{\#}(\beta)\right) .
$$




\subsection{Gamma Family}

Let $\Gamma(\alpha, 2 \beta), \alpha, \beta>0$, denote the Gamma variate with the p.d.f.

$$
f(x)=\frac{1}{\Gamma(\alpha)(2 \beta)^{\alpha}} x^{\alpha-1} e^{-\frac{1}{2 \beta} x}, \quad x \geq 0,
$$

where $\Gamma(\alpha)$ is the Gamma function. The Laplace transform of $f(x)$ is given by

$$
\phi_{f}(s)=\left(\frac{1}{1+2 \beta s}\right)^{\alpha}, \quad s>\operatorname{Re}(1 / 2 \beta) .
$$

\subsection{A Gamma Density of Integral Order}

When $\alpha$ is integer, say $\alpha=n \geq 1, f(x)$ is the $n$-fold convolution of the exponential density $e(1 / 2 \beta ; x)$. Hence, the sharp coefficients of $f(x)$ are given as

$$
f^{\#}=\operatorname{CONV}_{i=1}^{n}\left(e^{\#}(1 / 2 \beta)\right)
$$

\subsection{B Gamma Density of Non-integral Order}

When $\alpha$ is not integer, the problem is more involved. From (A.1.1.2) and (2.4.2),

$$
T_{f}^{\#}(u)=(1+\beta)^{-\alpha}(1-u)^{\alpha}\left(1-\frac{1-\beta}{1+\beta} u\right)^{-\alpha}, \quad|u|<1 .
$$

Note that $(1-\theta u)^{t}$ has the generalized binomial expansion

$$
(1-\theta u)^{t}=\sum_{n=0}^{\infty} a_{n}(\theta, t) u^{n} ; \quad a_{n}(\theta, t)=\left(\begin{array}{c}
t \\
n
\end{array}\right)(-\theta)^{n}, \quad n \geq 0 .
$$

The coefficients $a_{n}(\theta, t)$ are simplified, using the recursion $a_{n}(\theta, t)=\theta\left(1-\frac{1+t}{n}\right) a_{n-1}(\theta, t)$ with $a_{0}(\theta, t)=1$, as

$$
a_{n}(\theta, t)=\theta^{n} \prod_{k=1}^{n}\left(1-\frac{1+t}{k}\right), \quad n \geq 1 ; \quad a_{0}(\theta, t)=1 .
$$

Hence, the functions $(1-u)^{\alpha}$ and $\left(1-\frac{1-\beta}{1+\beta} u\right)^{-\alpha}$ are expanded as

$$
(1-u)^{\alpha}=\sum_{n=0}^{\infty} b_{n} u^{n} ; \quad b_{n}=\prod_{k=1}^{n}\left(1-\frac{1+\alpha}{k}\right), b_{0}=1
$$

and

$$
\left(1-\frac{1-\beta}{1+\beta} u\right)^{-\alpha}=\sum_{n=0}^{\infty} c_{n} u^{n} ; \quad c_{n}=\left(\frac{1-\beta}{1+\beta}\right)^{n} \prod_{k=1}^{n}\left(1-\frac{1-\alpha}{k}\right), c_{0}=1 .
$$

Let $\boldsymbol{b}=\left(b_{n}\right)$ and $\boldsymbol{c}=\left(c_{n}\right)$ with an appropriate truncation. It then follows from (2.4.4) that

$$
\boldsymbol{f}^{\#}=(1+\beta)^{-\alpha} \operatorname{CONV}(\boldsymbol{b}, \boldsymbol{c})
$$

Remark 2.4.1. Let $f_{1}(x)$ and $f_{2}(x)$ be the densities of $\Gamma\left(\alpha_{1}, \beta_{1}\right)$ and $\Gamma\left(\alpha_{2}, \beta_{2}\right)$, respectively. Suppose $0<\alpha_{1}<1 / 2$. It is easy to see that $f_{1}(x)$ is not square integrable. This means that the Laguerre transform does not exist for $f_{1}(x)$. The Laguerre sharp transform of $f_{1}(x)$ 
is, however, well defined via (2.4.9), see [19]. The sharp coefficients $\left(f_{n}^{\#}\right)_{0}^{\infty}$ of $f(x)=f_{1} * f_{2}(x)$ are also well defined through the convolution property of the Laguerre transform. If $f(x)$ becomes square integrable, one can evaluate $f(x)$ in terms of $\boldsymbol{f}^{\dagger}=\operatorname{PSUM}\left(\boldsymbol{f}^{\#}\right)$.

Remark 2.4.2. Two types of numerical difficulties may arise in handling Gamma densities. When $\alpha$ in (2.4.1) is too large, $b_{n}$ and $c_{n}$ in (2.4.7) and (2.4.8), respectively, become too large in the absolute value for computers to deal with. Also, when $\beta$ there is large or small so that the ratio $\left|\frac{1-\beta}{1+\beta}\right|$ becomes close to 1 , the sequence $\left(c_{n}\right)_{0}^{\infty}$ has a long tail, which in turn brings a storage problem. The latter problem can be solved by employing the scaling technique as described in Remark 2.3.1. The former may be solved by taking advantage of the divisibility of Gamma variates. That is, first obtain the sharp coefficients $\left(f_{M: n}^{\#}\right)_{0}^{\infty}$ of $\Gamma(\alpha / M, 2 \beta)$ by appropriately choosing a positive integer $M$ so that $\left(f_{M: n}^{\#}\right)_{0}^{\infty}$ can be obtained. One then may be able to calculate the sharp coefficients of $\Gamma(\alpha, \beta)$ by convolving $\left(f_{M: n}^{\#}\right)_{0}^{\infty}$ $M$ times with itself on the lattice.

\subsection{Log-beta Density}

Let $\beta(v, w)$ be the beta variate with the p.d.f.

$$
f_{\beta}(x)=\frac{\Gamma(v+w)}{\Gamma(v) \Gamma(w)} x^{v-1}(1-x)^{w-1}
$$

and let $U(v, w)=-\log \beta(v, w)$. The log-beta variate $U(v, w)$ is a useful tool for the asymptotic study of $\beta(v, w)$. The p.d.f. of $U(v, w)$ is given as

$$
f_{U}(x)=\frac{\Gamma(v+w)}{\Gamma(v) \Gamma(w)} e^{v x}\left(1-e^{-x}\right)^{w-1} .
$$

Let $w=[w]+\theta$, where $[w]$ is the largest integer less than or equal to $w$. Keilson and Sumita [17] showed that $f_{U}(x)$ is given as convolution of a completely monotone density $f_{1}(x)$ and a PF density $f_{2}(x)$. Stating their result more specifically, they obtained

$$
f_{1}(x)=\frac{\Gamma(v+w)}{\Gamma(v) \Gamma(w)} \sum_{k=0}^{\infty} p_{\theta k} e(k+v ; x) ; \quad p_{\theta k}=\frac{1}{k+v} \prod_{j=1}^{K}\left(1-\frac{\theta}{j}\right), p_{\theta 0}=1
$$

and

$$
f_{2}(x)=e(v+\theta ; x) * e(v+\theta+1 ; x) * \cdots * e(v+\theta+[w]-1 ; x) .
$$

It is seen from the convolution property that

$$
\boldsymbol{f}_{2}^{\#}=\operatorname{CONV}_{j=0}^{[w]-1}\left(e^{\#}(v+\theta+j)\right)
$$

and from the mixing property with an appropriate truncation, say at $K$, that

$$
\boldsymbol{f}_{1}^{\#}=\operatorname{MIX}\left(p_{\theta 0}, \cdots, p_{\theta K} ; \boldsymbol{e}^{\#}(v), \cdots, \boldsymbol{e}^{\#}(v+K)\right) .
$$

The sharp coefficients of $f(x)$ is then given as

$$
f_{U}^{\#}=\operatorname{CONV}\left(\boldsymbol{f}_{1}^{\#}, \boldsymbol{f}_{2}^{\#}\right)
$$




\subsection{Normal Family}

Let $N\left(\mu, \sigma^{2}\right)$ be the normal variate having the mean $\mu$ and the variance $\sigma^{2}$. Since shifting the mean is equivalent to shifting its density function, it suffices to consider $N\left(0, \sigma^{2}\right)$. When $\mu \neq 0$, we invoke (1.3.2) to get the sharp coefficients for $N\left(\mu, \sigma^{2}\right)$. Denote by $f(x)$ the p.d.f. of $N\left(0, \sigma^{2}\right)$, i.e.

$$
f(x)=\frac{1}{\sqrt{2 \pi} \sigma} e^{-\frac{x^{2}}{2 \sigma^{2}}}, \quad-\infty<x<\infty
$$

Clearly, $f(x)$ is symmetric about 0 . Let $g(x)=f(x) U(x)$. Then $f(x)=g(x) U(x)+$ $g(-x) U(-x)$, see $\S 2.2$. Thus, it is enough to obtain $g_{n}^{\dagger}$ or $g_{n}^{\#}$ for $n \geq 0$. If they are found, the Laguerre coefficients $f_{n}^{\#}$ for $-\infty<n<\infty$ are obtained by $\boldsymbol{f}^{\#}=\operatorname{CJCT}\left(\boldsymbol{g}^{\#}, \boldsymbol{g}^{\#}\right)$.

\subsection{A Normal Density}

Let $\phi_{g}(s)$ be the Laplace transform of $g(x)$. Then, it is easily seen that

$$
\phi_{g}(s)=e^{\sigma^{2} s^{2} / 2} \frac{1}{\sqrt{2 \pi} \sigma} \int_{s \sigma^{2}}^{\infty} e^{-x^{2} / 2 \sigma^{2}} d x
$$

It follows that

$$
\phi_{g}^{\prime}(s)-s \sigma^{2} \phi_{g}(s)+\frac{\sigma}{\sqrt{2 \pi}}=0 .
$$

Thus, by taking $v(s)=1, w(s)=-\sigma^{2} s$ and $z(s)=\sigma / \sqrt{2 \pi}$, one has the first order differential equation (2.1.2). Hence, from (2.1.4),

$$
(1-u)^{3} \frac{d}{d u} T_{g}^{\dagger}(u)-\left[\frac{\sigma^{2}}{2}(1+u)+(1-u)^{2}\right] T_{g}^{\dagger}(u)+\frac{\sigma}{\sqrt{2 \pi}}=0 .
$$

Comparing the coefficients of $u^{n}$, one then has

$$
g_{1}^{\dagger}=\left(1+\frac{\sigma^{2}}{2}\right) g_{0}^{\dagger}-\frac{\sigma}{\sqrt{2 \pi}}, \quad g_{2}^{\dagger}=\left(1+\frac{3 \sigma^{2}}{2}+\frac{\sigma^{4}}{8}\right) g_{0}^{\dagger}-\frac{1}{4}\left(8+\sigma^{2}\right) \frac{\sigma}{\sqrt{2 \pi}}
$$

and

$$
g_{n}^{\dagger}=\frac{1}{n}\left[\left(3 n-2+\frac{\sigma^{2}}{2}\right) g_{n-1}^{\dagger}-\left(3 n-4-\frac{\sigma^{2}}{2}\right) g_{n-2}^{\dagger}+(n-2) g_{n-3}^{\dagger}\right], n \geq 3
$$

Hence, once $g_{0}^{\dagger}$ is known, $g_{n}^{\dagger}$ for $n \geq 1$ can be evaluated recursively. For the value $g_{0}^{\dagger}$, one has

$$
g_{0}^{\dagger}=\int_{0}^{\infty} \ell_{0}(x) g(x) d x=\frac{e^{\sigma^{2} / 8}}{\sqrt{2 \pi}} \int_{\sigma / 2}^{\infty} e^{-\frac{y^{2}}{2}} d y
$$

and the value of the tail integral can be found in a table, see e.g. [2].

Remark 2.5.1. It is reported in [33] that relatively many terms of the Laguerre coefficients are required to guarantee accuracy for the normal density. For example, about 250 terms are needed to make the truncation error less than $10^{-10}$ for the 1 st and 2 nd moments of $N(0,1)$. If only 80 terms are taken, one is merely guaranteed by about 8 decimals of accuracy for the 0th moment, 6 decimals for the 1st moment and 3 decimals for 2 nd moment. The more terms are calculated, however, the more numerical errors are accumulated due to the nature of the forwardly solved recursion formula. Extensive numerical experiments 
show that this error is intolerable when $n \geq 80$. To overcome the difficulty, the following backwardly solving technique has been developed in Sumita [33].

Consider the backward recurrence formula for $g_{n}^{\dagger}$, i.e.

$$
(n+3) g_{n+3}^{\dagger}-\left(3 n+7+\frac{\sigma^{2}}{2}\right) g_{n+2}^{\dagger}+\left(3 n+5-\frac{\sigma^{2}}{2}\right) g_{n+1}^{\dagger}=(n+1) g_{n}^{\dagger}
$$

for $n \geq 0$. Define

$$
\boldsymbol{B}_{n+1}=\left(\begin{array}{ccc}
\frac{3 n+5-\frac{\sigma^{2}}{2}}{n+1} & \frac{3 n+7+\frac{\sigma^{2}}{2}}{n+1} & \frac{n+3}{n+1} \\
1 & 0 & 0 \\
0 & 1 & 0
\end{array}\right) ; \quad \boldsymbol{g}_{n+1}=\left(\begin{array}{c}
g_{n+1}^{\dagger} \\
g_{n+2}^{\dagger} \\
g_{n+3}^{\dagger}
\end{array}\right)
$$

for $n \geq 0$. Note that $\boldsymbol{B}_{n+1}$ is of full rank for all $n \geq 0$ and

$$
\boldsymbol{B}_{n+1} \boldsymbol{g}_{n+1}=\boldsymbol{g}_{n}, \quad n \geq 0 \text {. }
$$

By defining $\boldsymbol{M}_{N+1}=\boldsymbol{B}_{1} \cdots \boldsymbol{B}_{N+1}$, one has from (2.5.10) that

$$
M_{N+1} g_{N+1}=g_{0}
$$

which is known. Let $\boldsymbol{x}_{i}, i=1,2,3$, be any independent vectors on $R^{3}$ and let $\boldsymbol{y}_{i}=M_{N+1} \boldsymbol{x}_{i}$. Denote $\boldsymbol{X}=\left(\boldsymbol{x}_{1}, \boldsymbol{x}_{2}, \boldsymbol{x}_{3}\right)$ and $\boldsymbol{Y}=\left(\boldsymbol{y}_{1}, \boldsymbol{y}_{2}, \boldsymbol{y}_{3}\right)$, from which one has $\boldsymbol{Y}=\boldsymbol{M}_{N+1} \boldsymbol{X}$. Note that, since $\boldsymbol{M}_{N+1}$ is of full rank, $\boldsymbol{y}_{i}$ are linearly independent. Hence, there exists $\boldsymbol{\lambda}=$ $\left(\lambda_{1}, \lambda_{2}, \lambda_{3}\right)^{T}$ such that

$$
\boldsymbol{g}_{0}=\sum_{i=1}^{3} \lambda_{i} \boldsymbol{y}_{i}=\boldsymbol{Y} \boldsymbol{\lambda}=M_{N+1} \boldsymbol{X} \boldsymbol{\lambda}
$$

From $(2.5 .11),(2.5 .12)$ and nonsingularity of $M_{N+1}$, one concludes that

$$
\boldsymbol{g}_{N+1}=\boldsymbol{X} \boldsymbol{\lambda}=\boldsymbol{X} \boldsymbol{Y}^{-1} \boldsymbol{g}_{0}
$$

Hence, one has the following algorithm.

\section{Algorithm 2.5.2.}

(1) Choose a set of independent vectors $\boldsymbol{x}_{i}$ on $R^{3}, i=1,2,3$.

(2) Starting with $\boldsymbol{x}_{i}$, proceed the backward recursion formula (2.5.8) $N$ times, obtaining $\boldsymbol{y}_{i}$ for each $i$.

(3) Construct $\boldsymbol{X}$ and $\boldsymbol{Y}$ and evaluate $\boldsymbol{g}_{N+1}=\boldsymbol{X} \boldsymbol{Y}^{-1} \boldsymbol{g}_{0}$.

(4) Calculate $g_{n}^{\dagger}, 0 \leq n \leq N$, by (2.5.8) recursively.

The accuracy of the algorithm can be easily checked by comparing $g_{0}^{\dagger}$ obtained via the backward recurrence formula with the exact $g_{0}^{\dagger}$ given in (2.5.7).

\subsection{B Folded Normal Density}

Note that the folded normal density associated with $N\left(0, \sigma^{2}\right)$ is just $2 g(x)$.

\subsection{Rayleigh Density}


The p.d.f. of Rayleigh distribution is given by

$$
f(x)=\frac{x}{\sigma^{2}} e^{-\frac{x^{2}}{2 \sigma^{2}}}=\frac{\sqrt{2 \pi}}{\sigma} x g(x) .
$$

Hence, from (1.4.5), one has

$$
\boldsymbol{f}^{\#}=\frac{\sqrt{2 \pi}}{\sigma} \operatorname{MUL}\left(\boldsymbol{g}^{\#}\right)
$$

\subsection{Logistic Family}

\subsection{A Logistic Density}

Let $X$ be a logistic variate having the p.d.f.

$$
f(x)=\frac{\beta e^{-\beta x}}{\left(1+e^{-\beta x}\right)^{2}}, \quad-\infty<x<\infty, \quad \beta>0 .
$$

The bilateral Laplace transform of $f(x)$ is given by

$$
\phi_{f}(s)=\Gamma\left(1+\frac{s}{\beta}\right) \Gamma\left(1-\frac{s}{\beta}\right), \quad|R e(s)|<\beta .
$$

Let $Z_{k}$ be mutually independent random variables having the p.d.f.s $g_{k}(x)=\frac{\beta k}{2} e^{-\beta k|x|},-\infty<$ $x<\infty$, respectively. That is, each $g_{k}(x)$ is a Laplace-like density given in $§ 2.3 \mathrm{~F}$. Sumita $[33,34]$ showed that $X \stackrel{\mathrm{d}}{=} \sum_{k=1}^{\infty} Z_{k}$ ( $\stackrel{\mathrm{d}}{=}$ stands for equality in law). The sharp coefficients $\left(g_{k: n}^{\#}\right)_{-\infty}^{\infty}$ of $g_{k}(x)$ are available in $\S 2.3 \mathrm{~F}$. Hence, theoretically, the sharp coefficients $\left(f_{n}^{\#}\right)_{-\infty}^{\infty}$ of $f(x)$ can be obtained by convolving $\left(g_{k: n}^{\#}\right)_{-\infty}^{\infty}$ for $k \geq 1$. In practice, however, the numerical problem stated in Remark 2.3.1 arises. This difficulty was solved by the following idea, see Sumita $[33,34]$. Let

$$
X \stackrel{\mathrm{d}}{=} W_{1}+W_{2} ; \quad W_{1}=\sum_{k=1}^{M} Z_{k} \text { and } W_{2}=\sum_{k=M+1}^{\infty} Z_{k}
$$

where $M$ is an appropriately chosen positive integer. Denote by $\phi_{1}(s)$ the bilateral Laplace transform of the p.d.f. of $W_{1}$. Then, by the definition,

$$
\phi_{1}(s)=\prod_{k=1}^{M} \frac{(k / \beta)^{2}}{\left(\frac{k}{\beta}+s\right)\left(\frac{k}{\beta}-s\right)}=\sum_{k=1}^{M} a_{k} \frac{(k / \beta)^{2}}{\left(\frac{k}{\beta}+s\right)\left(\frac{k}{\beta}-s\right)},
$$

where

$$
a_{k}=\lim _{s \rightarrow-k / \beta}\left(\frac{k}{\beta}+s\right) \phi_{1}(s)=\frac{k}{2 \beta} \prod_{j=1, \neq k}^{M} \frac{j^{2}}{(j-k)(j+k)} .
$$

The sharp coefficients corresponding to $W_{1}$ are then obtained as

$$
\boldsymbol{f}_{1}^{\#}=\operatorname{MIX}\left(a_{1}, \cdots, a_{M} ; \boldsymbol{g}_{1}^{\#}, \cdots, \boldsymbol{g}_{M}^{\#}\right) .
$$

For the skinny part $W_{2}$, we approximate it by the normal variate $N\left(\mu, \sigma^{2}\right)$ of the same mean and variance. It is easy to see that

$$
\mu=E\left[W_{2}\right]=0 ; \quad \sigma^{2}=\operatorname{Var}\left[W_{2}\right]=\beta^{2}\left[\frac{\pi^{2}}{3}-2 \sum_{k=1}^{M} \frac{1}{k^{2}}\right] .
$$


A way of finding the Laguerre coefficients $\boldsymbol{f}_{2}^{\#}$ for $N\left(0, \sigma^{2}\right)$ was developed in $\S 2.5$. The approximate sharp coefficients $\boldsymbol{f}^{\#}$ of $f(x)$ in $(2.6 .1)$ are then obtained as $f^{\#}=\operatorname{CONV}\left(f_{1}^{\#}, f_{2}^{\#}\right)$.

Remark 2.6.1. No analytical result is available for the numerical error introduced by the normal approximation of $W_{2}$. The density function of both $W_{2}$ and $N\left(0, \sigma^{2}\right)$, however, are quite delta-like function for a modest value of $M$, say $M=20$. The approximation given here provides a surprising accuracy for the values of $f(x)$, see Sumita [33, 34].

\subsection{B Folded Logistic Density}

The folded logistic distribution has the density function $f_{F}(x)=2 f(x), x \geq 0$, where $f(x)$ is given in (2.6.1). The sharp coefficients $\left(f_{F: n}^{\#}\right)_{0}^{\infty}$ of $f_{F}(x)$ are obtained as

$$
f_{F: n}^{\#}=2 f_{n}^{\#}, \quad n \geq 1 ; \quad f_{F: 0}^{\#}=f_{0}^{\#},
$$

see $(2.2 .3)$.

\subsection{Bivariate Exponential Distributions}

A particularly important class of bivariate distributions is the class of exponential distributions. Several types of bivariate exponential distributions have been studied by many authors. The distributions introduced in the literature, however, have complicated forms and the absolute continuity with respect to the two dimensional Lebesgue measure is often lost. Accordingly, it is hard to obtain the Laguerre coefficients for bivariate exponential distributions in general form. Nevertheless, they are often found via numerical procedures as we shall see. In this section, following the work of Kijima [20], the procedures for obtaining the Laguerre coefficients for bivariate exponential distributions of importance are briefly described. For other distributions such as bivariate Gamma distributions of nonintegral order, bivariate logistic distributions and bivariate normal distributions, the reader is referred to Kijima [20].

\subsection{A Perfect Correlation}

Let $Z$ be an exponential random variable with mean 1 . Consider then a pair of random variables $(X, Y)$ defined by

$$
\begin{cases}X=a Z, & a>0 \\ Y=b Z, & b>0\end{cases}
$$

The bivariate random vector $(X, Y)$ has a bivariate exponential distribution of perfect correlation. Let $F(x, y)$ be the distribution function of $(X, Y)$. It should be noted that $F$ is not absolutely continuous. The corresponding Laplace-Stieltjes transform is given by

$$
\phi(s, w)=\int_{0}^{\infty} \int_{0}^{\infty} e^{-s x-w y} d F(x, y)=\frac{1}{1+a s+b w} .
$$

From the key formula (A.2.1.13) and (A.2.1.9), it can be seen that

$$
T^{\dagger}(u, v)=\sum_{m=0}^{\infty} \sum_{n=0}^{\infty} f_{m n}^{\dagger} u^{m} v^{n}=\frac{A+B}{2} \sum_{n=0}^{\infty}(A u+B v+C u v)^{n} .
$$

Here we set

$$
A=\frac{2-a+b}{2+a+b}, \quad B=\frac{2+a-b}{2+a+b} \text { and } C=\frac{a+b-2}{2+a+b}
$$


Let

$$
\mathrm{BEXP}: R^{2} \rightarrow R(0, M ; 0, N)
$$

be the bivariate lattice operator defined by

$$
\boldsymbol{A}=\operatorname{BEXP}(a, b) \Leftrightarrow a_{m n}=\frac{A+B}{2} \sum_{j=\max \{m, n\}}^{m+n} q(m, n ; j),
$$

where

$$
q(m, n ; j)=\frac{1}{A^{n} B^{m}}(\dot{m})\left(j \underline{m}_{m}\right) A^{j} B^{j} C^{m+n-j}
$$

for $0 \leq m, n \leq j \leq m+n$. Note that $q(m, n ; j)$ satisfy the recursion formulas

$$
q(m, n ; j+1)=q(m, n ; j) \frac{A B\left(\frac{m}{j-n}-1\right)}{C\left(1-\frac{m}{j+1}\right)\left(1+\frac{1}{j-n}\right)}
$$

and

$$
q(m, n+1 ; j+1)=q(m, n ; j) \frac{B}{\left(1-\frac{m}{j+1}\right)},
$$

with $q(0, n ; n)=B^{n}$ and $q(m, m ; m)=C^{m}$. It then follows from (2.7.5) that

$$
F^{\dagger}=\operatorname{BEXP}(a, b)
$$

Remark 2.7.1. Since $F(x, y)$ is not absolutely continuous, the dagger coefficients $\left(f_{m n}^{\dagger}\right)$ have no meaning. But, $\bar{F}(x, y)=\operatorname{Pr}[X>x, Y>y]$ is square integrable as long as $E[X Y]<$ $\infty$. Hence, the values of

$$
F(x, y)=1-\bar{F}(0, y)-\bar{F}(x, 0)+\bar{F}(x, y)
$$

can be evaluated via the coefficients $\operatorname{BSUV}\left(\operatorname{BDIFF}\left(\boldsymbol{F}^{\dagger}\right)\right)$, which are the dagger coefficients of $\bar{F}(x, y)$, see (1.6.3) and (1.6.16).

Remark 2.7.2. The coefficients $\left(a_{m n}\right)_{m, n=0}^{\infty}$ in (2.7.5) have a long tail especially when the parameters $a$ and $b$ are large or too small. As for the univariate case, it is desired to rescale variables so that both $a$ and $b$ are near 2 . Extensive numerical experiments suggest that the truncation points $M$ and $N$ would better be chosen in such a way that one truncation point is twice larger than the other. For example, when $a=b=1$, taking $M=22$ and $N=50$ assures the relative error for $\bar{F}(x, y)$ less than $10^{-10}$ in the real domain (see Kijima [20]).

\subsection{B Marshall and Olkin}

Consider a system consisting of two independent components subject to shocks that are always fatal. Three types of shocks will occur according to Poisson processes with parameters $\lambda_{1}, \lambda_{2}$ and $\lambda_{3}$, respectively. The first type of shocks applies to component 1 only and the second type applies to component 2 only, while the third type applies to both components. Because of the third type of shocks, lifetimes $X$ and $Y$ of the components 1 and 2 , respectively, are correlated. Let $Z_{i}$ be exponential random variables with mean $1 / \lambda_{i}$, $i=1,2,3$, respectively. It is then readily seen that the lifetimes of the two components are given by

$$
\left\{\begin{array}{l}
X=\min \left\{Z_{1}, Z_{3}\right\} \\
Y=\min \left\{Z_{2}, Z_{3}\right\}
\end{array}\right.
$$


The joint distribution of $(X, Y)$ is first considered by Marshall and Olkin [23]. The distribution contains a singular part along the line $x=y$. Let $\bar{F}(x, y)=\operatorname{Pr}[X>x, Y>y]$. It is easy to see that

$$
\bar{F}(x, y)=\exp \left(-\lambda_{1} x-\lambda_{2} y-\lambda_{3} \max \{x, y\}\right), \quad x, y>0 .
$$

The Laplace transform of $\bar{F}$ is given by

$$
\phi_{\bar{F}}(s, w)=\left[\frac{1}{\lambda_{1}+\lambda_{3}+s}+\frac{1}{\lambda_{2}+\lambda_{3}+w}\right]\left(\frac{1}{\lambda+s+w}\right)
$$

where $\lambda=\lambda_{1}+\lambda_{2}+\lambda_{3}$. The dagger coefficients corresponding to the Laplace-Stieltjes transform $(\lambda+s+w)^{-1}$ can be obtained from (2.7.9), say $G^{\dagger}=\frac{1}{\lambda} \operatorname{BEXP}(1 / \lambda, 1 / \lambda)$. The dagger coefficients corresponding to the Laplace transforms $\left(\lambda_{1}+\lambda_{3}+s\right)^{-1}$ and $\left(\lambda_{2}+\lambda_{3}+w\right)^{-1}$ are found as $e_{1}^{\dagger}=\frac{1}{\lambda_{1}+\lambda_{3}} \operatorname{EXP}\left(\lambda_{1}+\lambda_{3}\right)$ and $e_{2}^{\dagger}=\frac{1}{\lambda_{2}+\lambda_{3}} \operatorname{EXP}\left(\lambda_{2}+\lambda_{3}\right)$, respectively, see $\S 2.3 \mathrm{~A}$. These dagger coefficients are converted into the sharp coefficients by the operators DIFF and BDIFF, see (1.1.1) and (1.6.1) respectively. Then, applying the marginal convolution properties of the bivariate Laguerre transform, the sharp coefficients of the survival function $\bar{F}(x, y)$ in $(2.7 .12)$ are obtained as

$$
\begin{aligned}
\overline{\boldsymbol{F}}^{\#}=\operatorname{MCONV} & \left(\operatorname{DifF}\left(\boldsymbol{e}_{1}^{\dagger}\right), \operatorname{BDIFF}\left(\boldsymbol{G}^{\dagger}\right)\right) \\
& +\operatorname{MCONV}\left(\operatorname{DifF}\left(\boldsymbol{e}_{2}^{\dagger}\right), \operatorname{BDIFF}\left(\boldsymbol{G}^{\dagger}\right)^{T}\right)^{T},
\end{aligned}
$$

see (1.6.9) and (1.6.11).

\subsection{Freund}

In [5], Freund considered a reliability model where a system has two components, say 1 and 2, with the lifetimes $X$ and $Y$ having the exponential densities $e(\alpha ; x)$ and $e(\beta ; x)$, respectively. The lifetimes $X$ and $Y$ are dependent in that a failure of either component changes the parameter of the lifetime distribution of the other component. That is, when component 1 fails, the parameter for $Y$ becomes $\beta^{\prime}$. When component 2 fails, the parameter for $X$ becomes $\alpha^{\prime}$. There is no other dependence. Thus, the joint density function $f(x, y)$ of $(X, Y)$ is given by

$$
f(x, y)= \begin{cases}\alpha \beta^{\prime} e^{-\beta^{\prime} y-\left(\alpha+\beta-\beta^{\prime}\right) x}, & 0 \leq x<y, \\ \alpha^{\prime} \beta e^{-\alpha^{\prime} x-\left(\alpha+\beta-\alpha^{\prime}\right) y}, & 0 \leq y \leq x .\end{cases}
$$

It should be noted that the marginal distributions are exponential only when $\alpha^{\prime}=\beta^{\prime}=\alpha+\beta$. The Laplace transform of $f(x, y)$ is obtained as

$$
\phi_{f}(s, w)=\frac{1}{\alpha+\beta+s+w}\left(\frac{\alpha^{\prime} \beta}{\alpha^{\prime}+s}+\frac{\alpha \beta^{\prime}}{\beta^{\prime}+w}\right) .
$$

Hence the Laguerre coefficients $\left(f_{m n}^{\#}\right)_{m, n=0}^{\infty}$ of $f(x, y)$ can be calculated in the same manner as described in $\S 2.7 \mathrm{~B}$.

\subsection{Moran}

Moran [29] constructed a bivariate exponential distribution as the joint distribution of

$$
\left\{\begin{array}{l}
X=\frac{1}{2}\left(Z_{1}^{2}+Z_{2}^{2}\right) \\
Y=\frac{1}{2}\left(Z_{3}^{2}+Z_{4}^{2}\right)
\end{array}\right.
$$


where $Z_{i}, i=1, \cdots, 4$, are all standard normal random variables. Random vectors. $\left(Z_{1}, Z_{3}\right)$ and $\left(Z_{2}, Z_{4}\right)$ are mutually independent but each pair has a bivariate normal distribution with correlation $\rho$. The bivariate Laplace transform for $(X, Y)$ is easily found as

$$
\phi(s, w)=\frac{1}{(1+s)(1+w)-\rho^{2} s w} .
$$

Inverting (2.7.18), one sees its p.d.f. to be

$$
f(x, y)=\sum_{n=0}^{\infty} \rho^{2 n}\left\{\sum_{k=0}^{n} \frac{(-1)^{k}}{k !}(\Re) x^{k} e^{-x}\right\}\left\{\sum_{k=0}^{n} \frac{(-1)^{k}}{k !}(\Re) y^{k} e^{-y}\right\} .
$$

The correlation between $X$ and $Y$ is $\rho^{2}$. By following the same arguments as in $\S 2.7 \mathrm{~A}$, one can see that the Laguerre coefficients $\left(f_{m n}^{\dagger}\right)_{m, n=0}^{\infty}$ of $f(x, y)$ in $(2.7 .19)$ are given by (2.7.5) with

$$
A=B=\frac{3+\rho^{2}}{9-\rho^{2}} \text { and } C=-\frac{1-\rho^{2}}{9-\rho^{2}} \text {. }
$$

\subsection{E Gumbel}

Let $(X, Y)$ be a pair of correlated random variables having the joint distribution function

$$
F(x, y)=\left(1-e^{-\lambda_{1} x}\right)\left(1-e^{-\lambda_{2} y}\right)\left(1+\alpha e^{-\lambda_{1} x-\lambda_{2} y}\right),
$$

where $\lambda_{1}, \lambda_{2}>0$ and $-1 \leq \alpha \leq 1$. The marginal distributions are exponential with the parameters $\lambda_{1}$ and $\lambda_{2}$, respectively. The correlation is $\alpha / 4$. This bivariate exponential distribution is introduced in Gumbel [6]. It is readily seen that $F$ is absolutely continuous and the Laplace transform of the density function $f(x, y)$ is given by

$$
\begin{aligned}
\phi_{f}(s, w)= & (1+\alpha)\left(\frac{\lambda_{1}}{\lambda_{1}+s}\right)\left(\frac{\lambda_{2}}{\lambda_{2}+w}\right)-\alpha\left(\frac{2 \lambda_{1}}{2 \lambda_{1}+s}\right)\left(\frac{\lambda_{2}}{\lambda_{2}+w}\right) \\
& -\alpha\left(\frac{\lambda_{1}}{\lambda_{1}+s}\right)\left(\frac{2 \lambda_{2}}{2 \lambda_{2}+w}\right)+\alpha\left(\frac{2 \lambda_{1}}{2 \lambda_{1}+s}\right)\left(\frac{2 \lambda_{2}}{2 \lambda_{2}+w}\right) .
\end{aligned}
$$

Note that the bivariate function having the Laplace transform

$$
\phi_{g}(s, w)=\left(\frac{\lambda_{1}}{\lambda_{1}+s}\right)\left(\frac{\lambda_{2}}{\lambda_{2}+w}\right)
$$

is $e\left(\lambda_{1} ; x\right) e\left(\lambda_{2} ; y\right)$, so that the corresponding Laguerre coefficients are given by

$$
\operatorname{OUTP}\left(\operatorname{EXP}\left(\lambda_{1}\right), \operatorname{EXP}\left(\lambda_{2}\right)\right)
$$

see (1.5.2). Hence, since the bivariate Laguerre transform is linear, it follows from (2.7.22) that

$$
\begin{array}{r}
\boldsymbol{F}^{\dagger}=(1+\alpha) \operatorname{OUTP}\left(\operatorname{EXP}\left(\lambda_{1}\right), \operatorname{EXP}\left(\lambda_{2}\right)\right)-\alpha \operatorname{OUTP}\left(\operatorname{EXP}\left(2 \lambda_{1}\right), \operatorname{EXP}\left(\lambda_{2}\right)\right) \\
-\alpha \operatorname{OUTP}\left(\operatorname{EXP}\left(\lambda_{1}\right), \operatorname{EXP}\left(2 \lambda_{2}\right)\right)+\alpha \operatorname{OUTP}\left(\operatorname{EXP}\left(2 \lambda_{1}\right), \operatorname{EXP}\left(2 \lambda_{2}\right)\right)
\end{array}
$$




\section{Applications of the Laguerre Transform}

The power of the Laguerre transform has been placed in evidence through extensive computational studies of a variety of applied probability models. Originally, applications of the Laguerre transform were centered on statistical problems $[13,17,34]$. The success of the Laguerre transform, however, clearly demonstrated that the method could provide unusual computational tools for analyzing dynamic system behavior. This, in turn, motivated further theoretical studies to develop new transform results, which may previously be considered to be of limited value behind the Laplacian curtain. Combined with such theoretical studies and extensions of the formalism to the matrix [33,36] and the bivariate $[20,38]$ forms, the Laguerre transform has opened new paths in transient analysis of queueing systems $[12,15$, $16,18,32,39,41]$, the structural analysis of semi-Markov and related processes[26, 27, 44, 47], reliability theory $[11,12,38,40,42,48]$, birth-death processes $[20,35,37,45]$, and dynamic performability analysis of computer/communication and production systems [21, 24, 28, 25, $43,46]$, among others. In order to demonstrate the power of the method, we examine in this section how the Laguerre transform enables one to quantify the dynamic waiting time behavior of an M/G/1 queueing system with exhaustive server vacations. Recently, Takagi [49] has analyzed the time-dependent queue length process for various $M / G / 1$ vacation models including the exhaustive discipline. To the authors' best knowledge, however, the dynamic waiting time analysis presented here is new.

\subsection{M/G/1 Queueing System with Exhaustive Server Vacations}

We consider a single server queueing system where customers form a sequence of indepen dently and identically distributed (i.i.d.) positive random variables, independent of the Poisson arrival process, with common cumulative distribution function (c.d.f.) $S(x)=\operatorname{Pr}[S \leq x]$. Whenever the server becomes idle, he takes another vacation of random duration. This process continues until he finds at least one customer in the system upon his return from vacation. He then starts servicing until he becomes idle again. Random vacation periods constitute a sequence of i.i.d. positive random variables, independent of the Poisson arrival process and customers' service times, with common c.d.f. $V(x)=\operatorname{Pr}[V \leq x]$.

Such a queueing system is often called an $M / G / 1$ queueing system with exhaustive server vacations. For more general $\mathrm{G} / \mathrm{G} / 1$ queueing systems with exhaustive server vacations, Doshi [3] has shown that the ergodic waiting time can be expressed as an independent sum of the forward recurrence time of a vacation period and the ergodic waiting time of the same queueing system without server vacations. Subsequently this ergodic decomposition theorem has been extended by Keilson and Servi [14] to include Bernoulli vacation schedules. Shanthikumar and Sumita [32] has developed a similar ergodic decomposition theorem in a broader context of a modified Lindley process with replacement, which unifies the previous results as special cases and extends further to incorporate possible correlations. Apart from the ergodic analysis, little work has been done for quantifying the dynamic waiting time behavior. In what follows, we study this problem for the $M / G / 1$ case via the Laguerre transform. 


\subsection{Exploration of Dynamic Waiting Time Structure via the Laguerre Trans- form}

For studying the dynamic waiting time structure of an $M / G / 1$ queueing system with exhaustive server vacations, we introduce the following notation:

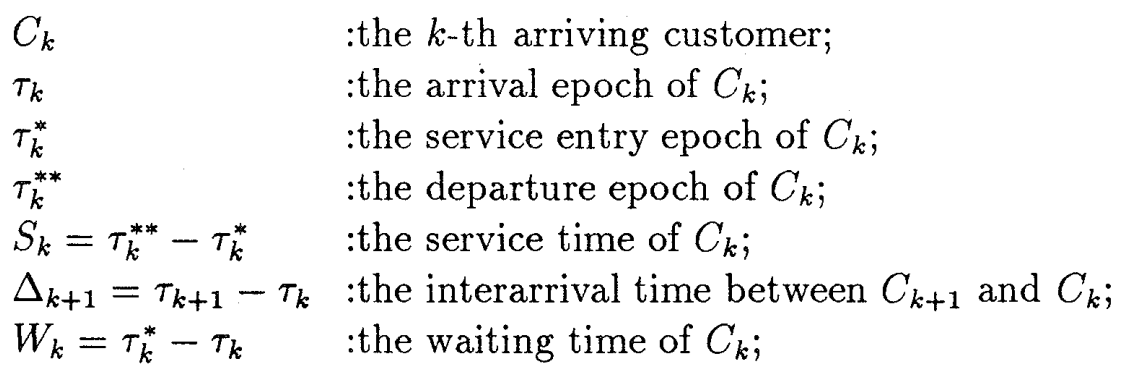

and, given $\tau_{k+1}>\tau_{k}^{* *}$,

$$
I_{k+1}=\tau_{k+1}-\tau_{k}^{* *}: \text { the idle period of the server given that } C_{k} \text { leaves him idle. }
$$

For convenience, we also define the survival function, the $n$-fold convolution and the LaplaceStieltjes transform corresponding to a c.d.f. $X(x)$ by:

$$
\begin{gathered}
\bar{X}(x)=1-X(x) ; \\
X^{(k)}(x)=\int_{0}^{x} X^{(k-1)}(x-y) d X(y), \quad k=1,2, \cdots ; \quad X^{(0)}(x)=U(x) ;
\end{gathered}
$$

and

$$
\phi_{X}(s)=\int_{0}^{\infty} e^{-s x} d X(x)
$$

Of particular interest is the waiting time structure of the queueing system characterized by the discrete time stochastic process $\left\{W_{k}: k=0,1,2, \cdots\right\}$. We assume that, at time $t=0, C_{0}$ has just arrived at the system and the server starts servicing him. Consequently, one has $W_{0}=0$. In the next theorem, we explicitly show that the stochastic process $\left\{W_{k}: k=0,1,2, \cdots\right\}$ can be viewed as a modified Lindley process with replacement.

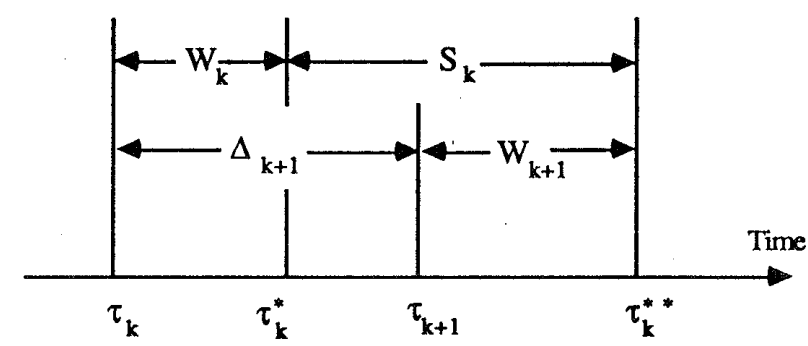

Figure 1 (a)

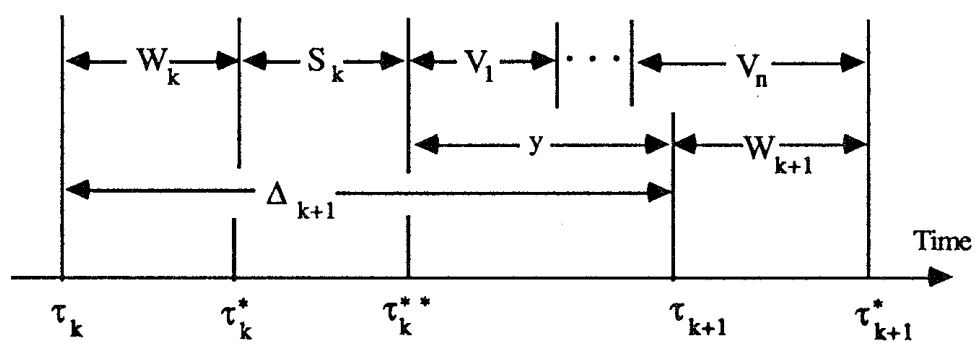

Figure $1(b)$ 
Theorem 3.2.1. Let $\xi_{k+1}=S_{k}-\Delta_{k+1}, k=0,1,2, \cdots$. Then one has

$$
W_{k+1}= \begin{cases}W_{k}+\xi_{k+1}, & W_{k}+\xi_{k+1} \geq 0 \\ R_{k+1}, & W_{k}+\xi_{k+1}<0\end{cases}
$$

where $R_{k+1}$ are i.i.d., independent of $W_{k}+\xi_{k+1}$, having a common c.d.f. $R(x)=\operatorname{Pr}[R \leq x]$ characterized by

$$
\phi_{R}(s)=\frac{\lambda}{\lambda-s}\left[1-\frac{1-\phi_{V}(s)}{1-\phi_{V}(\lambda)}\right] .
$$

Proof: It is clear from Figure 1(a) that $W_{k+1}=W_{k}+\xi_{k+1}$ if $W_{k}+\xi_{k+1} \geq 0$. Suppose $W_{k}+\xi_{k+1}<0$. Let $\left\{N_{V}(t): t \geq 0\right\}$ be the renewal process associated with a sequence of vacation periods $\left(V_{n}\right)_{n=1}^{\infty}$, and define the renewal function by $H_{V}(t)=E\left[N_{V}(t)\right]$. From the classical renewal theory, we recall that

$$
\int_{0}^{\infty} e^{-s t} d H_{V}(t)=\frac{\phi_{V}(s)}{1-\phi_{V}(s)}
$$

Let $\left\{\theta_{V}(t): t \geq 0\right\}$ be the stochastic process describing the residual lifetime of the renewal process at time $t$. One then sees from Figure 1(b) that $W_{k+1}=R_{\mid y}=\theta_{V}(y)$ if $I_{k+1}=y>0$. For evaluating the distribution $R(x \mid y)=\operatorname{Pr}\left[R_{\mid y} \leq x\right]=\operatorname{Pr}\left[\theta_{V}(y) \leq x\right]$, we first consider $\bar{F}_{n}(x, y)=\operatorname{Pr}\left[N_{V}(y)=n, \theta_{V}(y)>x\right]$. With notation $\delta_{n 0}=1$ if $n=0$ and $\delta_{n 0}=0$ if $n \neq 0$, it then follows that

$$
\bar{F}_{n}(x, y)=\delta_{n 0} \bar{V}(x+y)+\left(1-\delta_{n 0}\right) \int_{0}^{y} \bar{V}(x+y-z) d X^{(n)}(z) .
$$

Here the first term corresponds to the case of no renewal by time $y$. If so, the first vacation period must be greater than $(x+y)$ for $\theta_{V}(y)$ to be greater than $x$. If there have been $n$ renewals by time $t$, then the sum of the first $n$ yacation periods should be equal to $z$ with $z<y$, and the $(n+1)$-th vacation period must be greater than $(x+y-z)$ as described in the second term. By summing (3.2.7) over $n, n=0,1,2, \cdots$, one finds that

$$
\bar{R}(x \mid y)=\bar{V}(x+y)+\int_{0}^{y} \bar{V}(x+y-z) d H_{V}(z) .
$$

From (3.2.6) and (3.2.8), the Laplace-Stieltjes transform $\phi_{R}(s \mid y)=\int_{0}^{\infty} e^{-s x} d R(x \mid y)$ is given by

$$
\phi_{R}(s \mid y)=e^{s y}\left[\alpha_{V}(s, y)+\int_{0}^{y} \alpha_{V}(s, y-z) e^{-s z} d H_{V}(z)\right]
$$

where

$$
\alpha_{V}(s, y)=\int_{y}^{\infty} e^{-s x} d V(x) .
$$

Because of the Poisson arrivals of customers, given $\tau_{k+1}>\tau_{k}^{* *}, I_{k+1}$ are exponentially distributed with parameter $\lambda$. Hence, $\phi_{R}(s)=\int_{0}^{\infty} \lambda e^{-\lambda y} \phi_{R}(s \mid y) d y$, and the theorem follows from (3.2.9) and (3.2.10).

We next develop an iterative procedure for evaluating the distributions of $W_{k}$, assuming that the service time c.d.f. $S(x)$ and the vacation time c.d.f. $V(x)$ are absolutely continuous with corresponding p.d.f.s $s(x)$ and $v(x)$. The p.d.f. of the interarrival time is denoted by $e(\lambda ; x)$. The inputs to this procedure are the Laguerre sharp coefficients $s^{\#}=\left(s_{n}^{\#}\right)_{n=0}^{\infty}$, 
$\boldsymbol{e}^{\#}=\left(e_{-n}^{\#}(\lambda)\right)_{n=-\infty}^{0}$, and $\boldsymbol{v}^{\#}=\left(v_{n}^{\#}\right)_{n=0}^{\infty}$ corresponding respectively to the p.d.f.s $s(x), e(\lambda ; x)$ and $v(x)$. The Laguerre sharp coefficients $\boldsymbol{a}^{\#}=\left(a_{n}^{\#}\right)_{n=-\infty}^{\infty}$ corresponding to the p.d.f. $a(x)$ of $\xi_{k+1}$ are then obtained by

$$
\boldsymbol{a}^{\#}=\operatorname{CONV}\left(\boldsymbol{e}^{\#}, \boldsymbol{s}^{\#}\right) \text {. }
$$

Similarly, the Laguerre sharp coefficients $\boldsymbol{r}^{\#}=\left(r_{n}^{\#}\right)_{n=0}^{\infty}$ corresponding to the p.d.f. $r(x)$ of $R_{k+1}$ are given from (3.2.5) as

$$
\boldsymbol{r}^{\#}=\frac{\operatorname{CONV}\left(e^{\#}, \boldsymbol{s}^{\#}-\left(\delta_{n 0} \phi_{V}(\lambda)\right)_{n=0}^{\infty}\right)}{1-\phi_{V}(\lambda)}
$$

Here, the necessary constant $\phi_{V}(\lambda)$ should be obtained either directly from the Laplace transform $\phi_{V}(s)$ or from the identity

$$
\phi_{V}(\lambda)=T_{V}^{\#}(u) \text { at } u=\frac{2 \lambda-1}{2 \lambda+1} .
$$

Let $f_{k}(x)$ be the p.d.f. of $W_{k}$ with the Laguerre sharp coefficients $\boldsymbol{f}^{\#}(k)=\left(f_{n}^{\#}(k)\right)_{n=0}^{\infty}$. Similarly, let $f_{k+1}^{H}(x)$ be the p.d.f. of $W_{k}+\xi_{k+1}$ with $f^{H \#}(k+1)=\left(f_{n}^{H \#}(k+1)\right)_{n=-\infty}^{\infty}$. Although $f_{k+1}^{H}(x)$ has a full continuum support, the procedure developed in this section will require $f_{n}^{H \#}(k+1)$ only for $n \geq 0$, as we well see. It can be readily seen that

$$
f_{k+1}^{H}(x)=\int_{-\infty}^{\infty} f_{k}(x-y) a(y) d y
$$

and

$$
f_{k+1}(x)=f_{k+1}^{H}(x) U(x)+E_{k+1} r(x)
$$

where

$$
E_{k+1}=\int_{-\infty}^{0} f_{k+1}^{H}(x) d x=1+2 \sum_{n=0}^{\infty} f_{2 n+1}^{H \#}(k+1) .
$$

Equations (3.2.14) through (3.2.16) enable one to generate $\boldsymbol{f}^{\#}(k+1)$ from $\boldsymbol{f}^{\#}(k)$ recursively. This algorithm is now described below.

\section{Algorithm 3.2.2.}

Input: $\boldsymbol{s}^{\#}=\left(s_{n}^{\#}\right)_{n=0}^{\infty}, \boldsymbol{e}^{\#}=\left(e_{-n}^{\#}(\lambda)\right)_{n=-\infty}^{0}, \boldsymbol{v}^{\#}=\left(v_{n}^{\#}\right)_{n=0}^{\infty}$, and $\varepsilon>0$.

Output: $f^{\#}(k)=\left(f_{n}^{\#}(k)\right)_{n=0}^{\infty}$ for $k=1,2, \cdots$.

[0] Set $k=0$ and $\boldsymbol{f}^{\#}(0)=\left(\delta_{n 0}\right)_{n=0}^{\infty}$.

[1] Compute $\boldsymbol{a}^{\#}=\left(a_{n}\right)_{n=-\infty}^{\infty}$ and $\boldsymbol{r}^{\#}=\left(r_{n}^{\#}(k)\right)_{n=0}^{\infty}$ via (3.2.11) and (3.2.12), respectively.

[2] Loop: Find $\left(f_{n}^{H \#}(k+1)\right)_{n=0}^{\infty}$ from $\operatorname{CONV}\left(\boldsymbol{f}^{\#}(k), \boldsymbol{a}^{\#}\right)$.

[3] Compute $E_{k+1}$ via (3.2.16).

[4] Set $f_{0}^{\#}(k+1)=-\sum_{n=1}^{\infty} f_{n}^{H \#}(k+1)+E_{k+1} r_{0}^{\#}$.

[5] Set $f_{n}^{\#}(k+1)=f_{n}^{H \#}(k+1)+E_{k+1} r_{n}^{\#}, n \geq 1$.

[6] If $\left\|\boldsymbol{f}^{\#}(k)-\boldsymbol{f}^{\#}(k+1)\right\|>\varepsilon$, set $k=k+1$ and go back to Loop. 
[7] Stop.

We note that

$$
\sum_{n=0}^{\infty} f_{n}^{\#}(k+1)=T_{V}^{\#}(1)=\lim _{s \rightarrow \infty} \int_{0}^{\infty} e^{-s x} f_{k+1}(x) d x=0
$$

as it should be.

By evaluating the distributions of $W_{k}$ in a recursive manner, Algorithm 3.2.2 enables one to assess the speed of convergence to ergodicity. If only the ergodic waiting time distribution is needed, however, the Laplace transform permits to calculate it directly by convolving the residual vacation period distribution and the ergodic waiting time distribution of the same $\mathrm{M} / \mathrm{G} / 1$ system without vacations based on the Pollaczek-Khinchin formula.

Acknowledgments. Ushio Sumita is partially supported by the Nippon Telegraph and Telephone Research Fund. Masaaki Kijima is supported in part by General Scientific Research Grant (02680017) of the Ministry of Education, Science and Culture.

\section{References}

[A] Sumita, U. and Kijima, M. (1988), "Theory and Algorithms of the Laguerre Transform, Part I: Theory," Journal of the Operations Research Society of Japan, 31, 467-494.

[2] Abramowitz, M. and Stegun, I.A. (1965), Handbook of Mathematical Functions, Dover, New York.

[3] Doshi, B. (1985), "A Note on Stochastic Decomposition, in a GI/G/1 Queue with Vacations or Set-up Times," Journal of Applied Probability, 22, 419-428.

[4] Feller, W. (1971), An Introduction to Probability Theory and Its Applications, Vol.II, 2nd Ed., John Wiley, New York.

[5] Freund, R.J. (1961), "A Bivariate Extension of the Exponential Distribution," Journal of the American Statistical Association, 56, 971-977.

[6] Gumbel, E.J. (1958), "Distributions a Plusieurs Variables dont les Marges sont donnees," Comptes Rendus de l Academie des Sciences, Paris, 246, 2717-2720.

[7] Karlin, S. (1968), Total Positivity, Stanford University Press, Stanford, California.

[8] Karlin, S. and McGregor, J. (1957), "The Differential Equations of Birth and Death Processes and the Stieltjes Moment Problem," Transactions of the American Mathematical Society, 85, 489-546.

[9] Karlin, S. and McGregor, J. (1958), "Linear Growth Birth and Death Processes," Journal of Mathematics and Mechanics, 7, 643-662.

[10] Keilson, J. (1979), Markov Chain Models - Rarity and Exponentiality, Springer, New York.

[11] Keilson, J. and Nunn, W.R. (1979), "Laguerre Transformation as a Tool for the Numerical Solution of Integral Equations of Convolution Type," Applied Mathematics and Computation, 5, 313-359.

[12] Keilson, J., Nunn, W.R. and Sumita, U. (1981), "The Bilateral Laguerre Transform," Applied Mathematics and Computation, 8, 137-174. 
[13] Keilson, J., Petrondas, D. Sumita, U. and Wellner, J. (1983), "Significant Points for Some Tests of Uniformity on the Sphere," Journal of Statistical Computation and Simulation, 17, 195-218.

[14] Keilson, J. and Servi, L. (1986), "Oscillating Random Walk Models for G/G/1 Vacation Systems with Bernoulli Schedules," Journal of Applied Probability, 23, 790-802.

[15] Keilson, J. and Sumita, U. (1981), "Waiting Time Distribution Response to Traffic Surges via the Laguerre Transform," the Proceedings of the Conference on Applied Probability-Computer Science: The Interface, Boca Raton, Florida, 109-129.

[16] Keilson, J. and Sumita, U. (1983), "The Depletion Time for M/G/1 Systems and a Related Limit Theorem," Advances in Applied Probability, 15, 420-443.

[17] Keilson, J. and Sumita, U. (1983), "A Decomposition of the Beta Distribution, Related Order and Asymptotic Behavior," Annals of the Institute of Statistical Mathematics, Part A, 35, 243-253.

[18] Keilson, J. and Sumita, U. (1984), "Evaluation of the Total Time in System in a Preempt/Resume Priority Queue via a Modified Lindley Process," Advances in Applied Probability, 16, 840-856.

[19] Keilson, J. and Sumita, U. (1986), "A General Laguerre Transform and a Related Distance Between Probability Measures," Journal of Mathematical Analysis and Applications, 113, 288-308.

[20] Kijima, M. (1986), "Development of the Bivariate Laguerre Transform for Numerical Study of Bivariate Distributions and Bivariate Processes," Ph.D. Thesis, William E. Simon Graduate School of Business Administration, University of Rochester.

[21] Kubat, P., Sumita, U. and Masuda, Y. (1988), "Dynamic Performance Analysis of Communication/Computer Systems with Highly Reliable Components," Probability in the Engineering and Information Sciences, 2, 185-213.

[22] Ledermann, W. and Reuter, G.E.H. (1954), "Spectral Theory for the Differential Equations of Simple Birth and Death Processes," Philosophical Transactions of the Royal Society of London, Series A, 246, 321-369.

[23] Marshall, A.W. and Olkin, I. (1967), "A Multivariate Exponential Distribution," Journal of the American Statistical Association, 56, 971-977.

[24] Masuda, Y. (1987), "Dynamic Analysis of Computer and Communication Systems: Semi-Markov Approach," Ph.D. Thesis, William E. Simon Graduate School of Business Administration, University of Rochester.

[25] Masuda, Y., Shanthikumar, J.G. and Sumita, U. (1986), "A General Software Availability/Reliability Model: Numerical Exploration via the Matrix Laguerre Transform," Stochastic Models, 2, 203-236.

[26] Masuda, Y. and Sumita, U. (1987), "Analysis of Counting Process Associated with Semi-Markov Process: Number of Entries into a Subset of State Space," Advances in Applied Probability, 19, 767-783.

[27] Masuda, Y. and Sumita, U. (1990), "A Multivariate Reward Process Defined on a Semi-Markov Process and Its First Passage Time Distributions," Journal of Applied Probability, to appear. 
[28] Masuda, Y. and Sumita, U. (1989), "Numerical Analysis of Gracefully Degrading FaultTolerant Computer Systems: Semi-Markov Process and Laguerre Transform Approach," Working Paper Series No.QM89-13, William E. Simon Graduate School of Business Administration, University of Rochester.

[29] Moran, P.A.P. (1967), "Testing for Correlation between Nonnegative Variables," Biometrika, 54, 385-394.

[30] Neuts, S. (1981), Matrix-geometric Solutions in Stochastic Models - An Algorithmic Approach, Johns Hopkins University Press, Baltimore.

[31] Shanthikumar, J.G. (1985), "Bilateral Phase-type Distributions," Naval Research Logistic Quarterly, 32, 119-136.

[32] Shanthikumar, J.G. and Sumita, U. (1989), "Modified Lindley Process with Replacement: Dynamic Behavior, Asymptotic Decomposition and Applications," Journal of Applied Probability, 26, 552-565.

[33] Sumita, U. (1981), "Development of the Laguerre Transform Method for Numerical Exploration of Applied Probability Models," Ph.D. Thesis, William E. Simon Graduate School of Business Administration, University of Rochester.

[34] Sumita, U. (1983), "On Sums of Independent Logistic and Folded Logistic Variants Structural Tables and Graphs," Journal of Statistical Computation and Simulation, 17, 251-274.

[35] Sumita, U. (1984), "On Conditional Passage Time Structure of Birth-death Processes," Journal of Applied Probability, 21, 10-21.

[36] Sumita, U. (1984), "The Matrix Laguerre Transform," Applied Mathematics and Computation, 15, 1-28.

[37] Sumita, U. (1987), "On Limiting Behavior of Ordinary and Conditional First Passage Times for a Class of Birth-death Processes," Journal of Applied Probability, 24, 235-240.

[38] Sumita, U. and Kijima, M. (1985), "The Bivariate Laguerre transform and Its Applications - Numerical Exploration of Bivariate Processes," Advances in Applied Probability, 17, 683-708.

[39] Sumita, U. and Kijima, M. (1986), "On Optimal Bulk Size of Single-server Bulk-arrival Queueing Systems with Set-up Times: Numerical Exploration via the Laguerre Transform," Selecta Statistica Canadiana, 7, 77-108.

[40] Sumita, U. and Kijima, M. (1986), "Evaluation of Minimum and Maximum of a Correlated Pair of Random Variables via the Bivariate Laguerre Transform," Stochastic Models, 2, 123-149.

[41] Sumita, U. and Kijima, M. (1987), "Numerical Exploration of a Bivariate Lindley Process via the Bivariate Laguerre transform," Annals of Operations Research, 8, 321-349.

[42] Sumita, U. and Kijima, M. (1989), "The Laguerre transform of Product of Functions," Journal of Operations Research Society of Japan, 32, 279-285.

[43] Sumita, U. and Masuda, Y. (1986), "Analysis of Software Availability/Reliability Under the Influence of Hardware Failure," IEEE Transactions on Software Engineering: Special Issue on Software Reliability, 1, 32-41.

[44] Sumita, U. and Masuda, Y. (1987), "An Alternative Approach to the Analysis of Finite Semi-Markov and Related Processes," Stochastic Models, 3, 67-87. 
[45] Sumita, U. and Masuda, Y. (1987), "Classes of Probability Density Functions Having Laplace Transforms with Negative Zeros and Poles," Advances in Applied Probability, 19, 632-651.

[46] Sumita, U., Masuda, Y. and Kubat, P. (1989), "Development of Performance Functions for Communication/Computer Systems and Its Application to Dynamic Performance Analysis," Microelectronics and Reliability, 29, 973-991.

[47] Sumita, U. and Rieders, M. (1988), "First Passage Times and Lumpability of SemiMarkov Processes," Journal of Applied Probability, 25, 675-687.

[48] Sumita, U. and Shanthikumar, J.G. (1985), "A Class of Correlated Cumulative Shock Models," Advances in Applied Probability, 17, 133-147.

[49] Takagi, H. (1990), "Time-dependent Analysis of M/G/1 Vacation Models with Exhaustive Service," Queueing Systems, 6, 369-390.

[50] Widder, S. (1946), The Laplace Transform, Princeton University Press, Princeton.

Masaaki KIJIMA

Graduate School of Systems Management

University of Tsukuba, Tokyo

3-29-1 Otsuka, Bunkyo-ku, 112 Tokyo, Japan 